Virginia Commonwealth University vCU Scholars Compass

1996

\title{
Condensation of supersaturated vapors of hydrogen bonding molecules: Ethylene glycol, propylene glycol, trimethylene glycol, and glycerol
}

David B. Kane

Virginia Commonwealth University

M. Samy El-Shall

Virginia Commonwealth University, mselshal@vcu.edu

Follow this and additional works at: http://scholarscompass.vcu.edu/chem_pubs

Part of the Chemistry Commons

Kane, D., and El-Shall, M. S. Condensation of supersaturated vapors of hydrogen bonding molecules: Ethylene glycol, propylene glycol, trimethylene glycol, and glycerol. The Journal of Chemical Physics, 105, 7617 (1996). Copyright (C) 1996 American Institute of Physics.

\section{Downloaded from}

http://scholarscompass.vcu.edu/chem_pubs/45

This Article is brought to you for free and open access by the Dept. of Chemistry at VCU Scholars Compass. It has been accepted for inclusion in Chemistry Publications by an authorized administrator of VCU Scholars Compass. For more information, please contact libcompass@vcu.edu. 


\title{
Condensation of supersaturated vapors of hydrogen bonding molecules: Ethylene glycol, propylene glycol, trimethylene glycol, and glycerol
}

\author{
David Kane and M. Samy El-Shalla) \\ Department of Chemistry, Virginia Commonwealth University, Richmond, Virginia 23284-2006
}

(Received 2 May 1996; accepted 10 July 1996)

\begin{abstract}
The critical supersaturations required for the homogeneous nucleation (rate of $1 \mathrm{drop} \mathrm{cm}^{-3} \mathrm{~s}^{-1}$ ) of ethylene glycol, propylene glycol, trimethylene glycol and glycerol vapors have been measured over wide temperature ranges (e.g., 280-400 K) using an upward thermal diffusion cloud chamber. At lower temperatures the experimental nucleation rates are much higher than the predictions of the classical nucleation theory. Glycerol shows the best agreement between experiment and theory in the temperature range of 340-370 K. An apparent increase in the critical supersaturation of glycerol is observed with increasing carrier gas (helium) pressure and this effect is more pronounced at lower temperatures. The results from corresponding states and scaled nucleation models indicate that the nucleation behavior of glycerol is quite different from other glycols. Glycerol requires higher critical supersaturations compared to the other glycols at the same reduced temperatures. This implies quite small critical clusters for glycerol (20-50 molecules) in the temperature range 300-380 K. The discrepancy between experiment and theory at lower temperatures may be explained by considering that the surface tension of the critical clusters is lower than the bulk surface tension. It is, however, surprising that a Tolman type correction for the curvature dependent surface tension could be applicable for such small critical clusters. Further theoretical work is required in order to fully understand the observed higher nucleation rates at lower temperatures of glycols and glycerol. (C) 1996 American Institute of Physics. [S0021-9606(96)51139-6]
\end{abstract}

\section{INTRODUCTION}

Nucleation is one of the most ubiquitous and important phenomena in science and technology. It plays a role in materials science and metallurgy, crystal growth and chemical processing, aerosol formation, cavitation, crack propagation, quality control, turbine design, wind tunnels, atmospheric science, and cosmochemistry. ${ }^{1-4}$

Vapor phase homogeneous nucleation involves the decay of a metastable state, i.e., a supersaturated vapor, by the spontaneous occurrence of large thermal fluctuations, that is, through the formation of droplets of the liquid phase. Droplets that are larger than a critical size grow, and thus the stable phase results. The supersaturation at which the metastable state collapses and the nucleation rate increases explosively is known as the critical supersaturation. The experimental studies of homogeneous nucleation are usually concerned with two fundamental measurements. These are the determination of the temperature dependence of the critical supersaturation for the onset of nucleation and the determination of the temperature and supersaturation dependences of the rate of nucleation.,

There is currently an enormous growth of interest in studying the the vapor phase homogeneous nucleation for different classes of substances. ${ }^{5-22}$ This interest is due in part to the need for comparing experimental data with predictions from different nucleation theories in order to develop an adequate quantitative theory. In addition, these measurements are important in determining the nucleating ability of different vapors and the conditions of their participation in the formation of ultrafine aerosols.

a) Author to whom correspondence should be addressed.
The most popular theory of homogeneous nucleation of a liquid from its vapor is known as the classical nucleation theory $(\mathrm{CNT}) .{ }^{1-4}$ This theory is based on the capillarity approximation which treats the nucleus for condensation as a small fragment of a bulk liquid with the same macroscopic properties such as surface tension and density. Although the CNT has provided significant progress in qualitatively understanding the factors that control the formation of the new phase, it fails to provide a consistent molecular picture of the nucleation process as well as quantitative predictions of the rate of nucleation as a function of temperature and supersaturation. Several models have been suggested to improve the theory, but in most cases, they have retained the capillarity approximation. ${ }^{23-28}$ Other significant developments based on density functional theory, kinetic molecular theory, statistical mechanics, and molecular simulation methods have been proposed in recent years. ${ }^{29-44}$ Several useful reviews on the recent developments of nucleation theories are available in the literature..$^{35,43,44}$

Systematic studies of homogeneous nucleation of different classes of compounds with a variety of molecular properties are desirable in order to test nucleation theories. Such studies can also provide insight into the role of different molecular properties in the nucleation process. This information is necessary in order to develop scaling laws for homogeneous nucleation which are not dependent on any particular form of the theory. In this way, general patterns in nucleation behavior can be revealed that cannot be seen on the basis of a substance-by-substance comparison between experiment and theory. Toward this goal, we have examined the homogeneous nucleation of the nonpolar substances $\mathrm{SiCl}_{4}, \mathrm{SnCl}_{4}$, and $\mathrm{TiCl}_{4} \cdot{ }^{12-14}$ In these systems, the experi- 
mental critical supersaturations $\left(S_{c}\right)$ required for the onset of nucleation (rate of $1 \mathrm{drop} / \mathrm{cm}^{3} / \mathrm{s}$ ) agree within a few percent with the predictions of the CNT. However, when the experimental results are compared among the series using a suitable dimensionless form, it becomes clear that $\mathrm{TiCl}_{4}$ shows a different behavior compared to the other tetrachlorides $\mathrm{CCl}_{4}$, $\mathrm{SiCl}_{4}$, and $\mathrm{SnCl}_{4}$. We have also studied the homogeneous nucleation of the highly polar substances, acetonitrile, benzonitrile, nitromethane, and nitrobenzene where the dipole moments range from 3.5-4.4 D. ${ }^{9,10}$ The experimental results of this series reveal significant disagreements with the predictions of the CNT. The supersaturated vapors of these compounds are found to be more stable with respect to condensation than those of the weakly polar or nonpolar substances which are well described by the CNT.

In the present study, we investigate the vapor phase homogeneous nucleation of a series of hydrogen bonding substances, namely ethylene glycol, propylene glycol, trimethylene glycol and glycerol. These substances are characterized by strong hydrogen bonding interactions in the liquid phase and a strong tendency to supercool and vitrify at low temperatures into rigid glasses. They are also convenient media for various spectroscopic studies of biological molecules and they play important roles (particularly glycerol) in biological systems. Furthermore, these compounds are common working fluids in many technological processes, and it is important to measure the conditions (temperature, pressure, supersaturation) under which liquid droplets can be generated by homogeneous nucleation and growth from their supersaturated vapors.

The objectives of this work are to report the experimental data for the temperature dependence of the critical supersaturations of glycols and glycerol and to compare the results with the predictions of the CNT. Furthermore, we also analyze the experimental results in terms of corresponding states and scaling models for homogeneous nucleation in order to allow comparison with other structurally related substances.

\section{EXPERIMENT}

The present data were obtained using an upward thermal diffusion cloud chamber [see Fig. 1(a)]. The general description of this device and the details of its operation are available elsewhere. ${ }^{9}$ The onset of nucleation is determined by observing the forward scattering of light from drops falling through a horizontal $\mathrm{He}-\mathrm{Ne}$ laser beam positioned near the middle of the chamber. These drops originate near the elevation at which the maximum (peak) supersaturation occurs $(\sim 0.7$ reduced height). A measurement consists of setting the temperatures of the top and bottom plates so that the chamber is barely nucleating at a rate of $1-3$ drops $\mathrm{cm}^{-3} \mathrm{~s}^{-1}$. Under these conditions, measurements are made of the total pressure $P_{t}$, the temperature of the evaporating pool $T_{0}$, and the temperature of the upper plate $T_{1}$. The temperatures of both plates are then decreased (or increased) by about $5{ }^{\circ} \mathrm{C}$ and the measurement is repeated. To insure reproducibility each measurement was repeated at least three times. In order to eliminate the interference from ion nucleation resulting
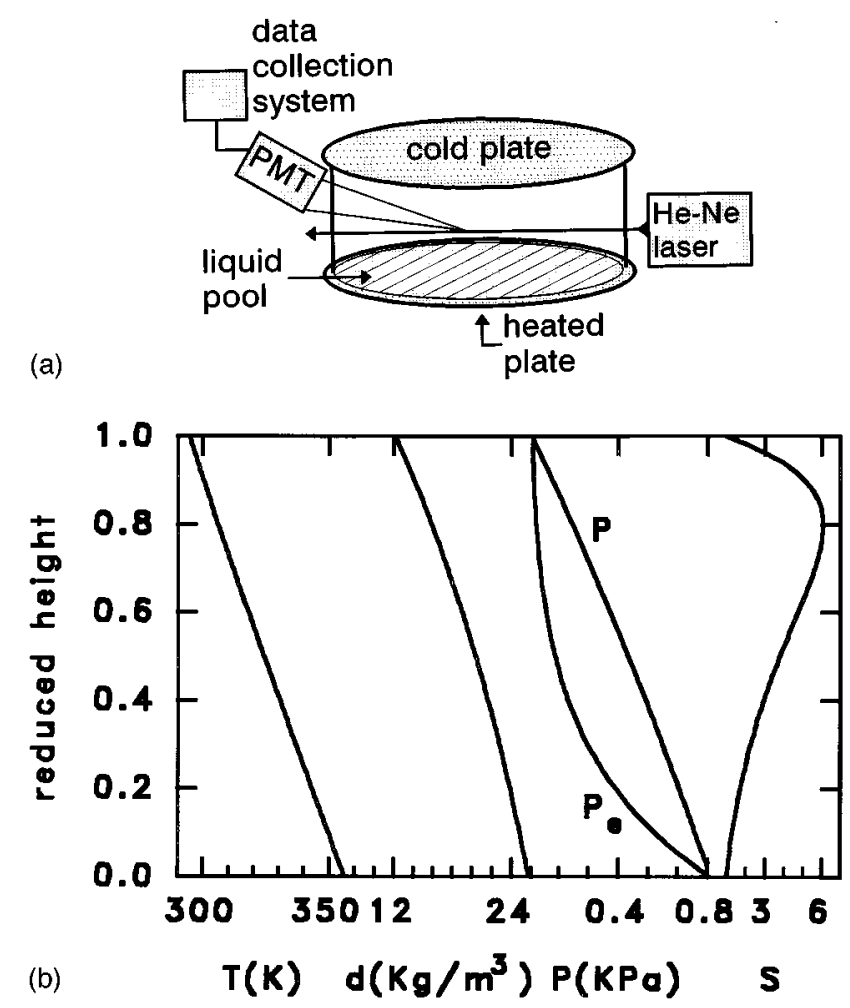

FIG. 1. (a) Schematic of the diffusion cloud chamber experiment for homogeneous nucleation. (b) Total vapor density, temperature, equilibrium vapor pressure, partial pressure and supersaturation profiles calculated for the observed nucleation of 1-3 drops $\mathrm{cm}^{-3} \mathrm{~s}^{-1}$ for ethylene glycol.

from cosmic ray or natural radioactive sources, the measurements are performed with a constant electric field of $40 \mathrm{~V} / \mathrm{cm}$ applied between the chamber plates.

All chemicals were obtained from Aldrich with stated purity of at least $99 \%$ and were degassed repeatedly by the freeze-pump-thaw method and transferred to the cloud chamber through a vacuum line. The carrier gas was research grade helium ( $99.999 \%$ pure).

\section{RESULTS AND DISCUSSION}

The chamber parameters $T_{0}, T_{1}$, and $P_{t}$ at which a steady nucleation rate of $1-3$ drops $\mathrm{cm}^{-3} \mathrm{~s}^{-1}$ occurs for ethylene glycol, propylene glycol, trimethylene glycol, and glycerol are listed in Tables I-IV, respectively. In all the experiments reported in Tables I-IV, no visible convection was observed in the chamber and the total vapor density was a monotonically decreasing function of chamber height.

The thermophysical properties needed to solve the boundary value problem associated with heat and mass flux in the chamber to obtain the supersaturation and temperature profiles are given in Table V. ${ }^{45-49}$ Based on the estimated errors in the thermophysical properties and the uncertainty in measuring $T_{0}, T_{1}$, and $P_{t}$, we estimate the overall error in the experimentally determined supersaturation as $4 \%-6 \%$.

An example of the dependence of supersaturation, temperature and partial pressure on elevation within the chamber is given in Fig. 1(b). The peak supersaturations achieved in the chamber as a function of temperature are plotted for each 
TABLE I. Measured data for each experiment for ethylene glycol. $T_{0}$, the temperature of the pool surface in Kelvin; $T_{1}$, the temperature of the top plate in Kelvin; $P_{t}$, the total pressure in $\mathrm{kPa} ; S_{\max }$, the maximum supersaturation in the chamber; $T_{\left(S_{\max }\right)}$, the temperature at $S_{\max }$, and $P_{t} / P_{0}$ is the pressure ratio in the chamber where $P_{0}$ is the equilibrium vapor pressure of ethylene glycol at $T_{0}$.

\begin{tabular}{|c|c|c|c|c|c|}
\hline$P_{\text {tot }}$ & $T_{0}$ & $T_{1}$ & $T\left(S_{\max }\right)$ & $S_{\max }$ & $P_{\text {tot }} / P_{0}$ \\
\hline 7.184 & 349.5 & 289.3 & 300.7 & 6.2 & 12.8 \\
\hline 7.251 & 356.0 & 294.7 & 306.0 & 6.1 & 8.9 \\
\hline 22.179 & 363.4 & 298.5 & 310.5 & 6.1 & 18.2 \\
\hline 7.744 & 365.3 & 302.7 & 314.5 & 5.8 & 5.8 \\
\hline 7.024 & 333.0 & 275.0 & 285.5 & 7.1 & 34.0 \\
\hline 7.238 & 339.8 & 280.7 & 291.8 & 6.7 & 22.9 \\
\hline 7.318 & 348.2 & 287.6 & 299.2 & 6.5 & 14.0 \\
\hline 7.011 & 332.3 & 274.6 & 285.0 & 7.0 & 35.5 \\
\hline 7.238 & 342.6 & 282.8 & 293.9 & 6.7 & 19.4 \\
\hline 6.891 & 336.0 & 277.7 & 288.7 & 6.8 & 27.6 \\
\hline 3.506 & 330.2 & 273.4 & 283.9 & 7.1 & 20.3 \\
\hline 3.546 & 337.4 & 279.5 & 290.5 & 6.9 & 13.0 \\
\hline 3.199 & 331.8 & 274.8 & 285.2 & 7.1 & 16.7 \\
\hline 3.452 & 344.9 & 285.9 & 296.6 & 6.7 & 8.0 \\
\hline 3.825 & 350.3 & 290.3 & 301.1 & 6.5 & 6.5 \\
\hline 21.953 & 351.3 & 287.8 & 299.3 & 6.8 & 35.2 \\
\hline 21.100 & 344.3 & 284.1 & 295.8 & 6.3 & 50.9 \\
\hline 22.539 & 362.1 & 298.3 & 310.8 & 5.8 & 19.9 \\
\hline 22.379 & 361.7 & 298.0 & 310.5 & 5.8 & 20.2 \\
\hline 38.401 & 359.2 & 294.2 & 306.7 & 6.3 & 39.6 \\
\hline 42.440 & 376.9 & 309.8 & 322.9 & 5.4 & 17.4 \\
\hline 40.933 & 362.7 & 298.2 & 310.5 & 5.8 & 34.9 \\
\hline 42.919 & 375.1 & 309.0 & 322.0 & 5.3 & 19.3 \\
\hline 59.927 & 382.4 & 314.7 & 328.0 & 5.1 & 18.8 \\
\hline 57.021 & 361.5 & 296.0 & 308.7 & 6.2 & 51.9 \\
\hline 58.181 & 367.5 & 301.1 & 313.9 & 5.9 & 38.5 \\
\hline 59.367 & 373.8 & 306.5 & 319.3 & 5.6 & 28.4 \\
\hline 69.364 & 404.9 & 333.0 & 347.7 & 4.7 & 7.8 \\
\hline 67.511 & 393.8 & 322.9 & 337.1 & 5.1 & 12.4 \\
\hline 76.442 & 417.8 & 344.0 & 359.0 & 4.4 & 5.0 \\
\hline 78.201 & 421.0 & 347.4 & 362.5 & 4.2 & 4.5 \\
\hline 62.633 & 380.2 & 312.8 & 326.1 & 5.1 & 21.8 \\
\hline 66.205 & 394.3 & 323.3 & 337.5 & 5.1 & 11.9 \\
\hline 73.843 & 380.9 & 312.2 & 325.9 & 5.3 & 24.9 \\
\hline 75.482 & 388.2 & 318.6 & 332.2 & 5.1 & 18.0 \\
\hline 79.507 & 401.3 & 330.0 & 344.2 & 4.7 & 10.4 \\
\hline 71.310 & 374.2 & 306.6 & 319.5 & 5.6 & 33.5 \\
\hline 74.602 & 386.1 & 316.6 & 330.3 & 5.2 & 19.6 \\
\hline 109.005 & 379.4 & 310.0 & 323.4 & 5.6 & 39.5 \\
\hline 113.470 & 390.8 & 320.5 & 334.5 & 4.9 & 23.9 \\
\hline 125.093 & 416.5 & 341.5 & 357.0 & 4.5 & 8.6 \\
\hline 109.031 & 379.7 & 310.2 & 323.6 & 5.6 & 38.9 \\
\hline 111.124 & 385.1 & 315.2 & 329.2 & 5.2 & 30.6 \\
\hline 113.430 & 391.1 & 320.0 & 334.1 & 5.1 & 23.6 \\
\hline 116.989 & 400.7 & 328.3 & 343.1 & 4.8 & 15.8 \\
\hline 130.731 & 402.5 & 329.3 & 344.3 & 4.8 & 16.3 \\
\hline 133.930 & 408.8 & 334.5 & 349.5 & 4.7 & 12.7 \\
\hline
\end{tabular}

experiment in Figs. 2-5 for ethylene glycol, propylene glycol, trimethylene glycol, and glycerol, respectively. The envelope of each set of peaks represents the variation of the critical supersaturation $\left(S_{c}\right)$ for each compound with temperature. These envelopes are shown as the solid lines in Figs. 2-5.

Because of the low vapor pressures of the studied compounds, it was not possible to examine the effect of carrier gas pressure on the nucleation rate over a wide temperature range without introducing convection effects which limit the window of stable operation of the cloud chamber. Therefore, measurements were carried out at different carrier gas pressures only over a limited temperature range. In these experiments, a small and reproducible increase in the critical supersaturation was observed with an increase in the carrier gas (helium) pressure. The pressure effect was more pronounced at lower temperatures and was most significant for glycerol in comparison to the three glycol compounds. An example of 
TABLE II. Measured data for each experiment for propylene glycol. $T_{0}$, the temperature of the pool surface; $T_{1}$, the temperature of the top plate in Kelvin; $P_{t}$, the total pressure in $\mathrm{kPa} ; S_{\max }$, the maximum supersaturation in the chamber; $T_{\left(S_{\max }\right)}$, the temperature at $S_{\max }$, and $P_{t} / P_{0}$ is the pressure ratio in the chamber where $P_{0}$ is the equilibrium vapor pressure of propylene glycol at $T_{0}$.

\begin{tabular}{|c|c|c|c|c|c|}
\hline$P_{\text {tot }}$ & $T_{0}$ & $T_{1}$ & $T\left(S_{\max }\right)$ & $S_{\max }$ & $P_{\text {tot }} / P_{0}$ \\
\hline 21.966 & 342.9 & 289.6 & 301.3 & 4.1 & 35.7 \\
\hline 22.406 & 348.0 & 294.3 & 306.4 & 3.9 & 27.0 \\
\hline 21.593 & 342.9 & 289.5 & 301.1 & 4.1 & 35.0 \\
\hline 22.219 & 348.3 & 294.4 & 306.5 & 3.9 & 26.5 \\
\hline 23.619 & 354.8 & 299.9 & 312.0 & 3.8 & 19.6 \\
\hline 23.419 & 360.6 & 304.8 & 317.4 & 3.8 & 14.2 \\
\hline 23.979 & 367.0 & 310.1 & 322.7 & 3.8 & 10.4 \\
\hline 24.565 & 372.4 & 315.0 & 327.4 & 3.7 & 8.2 \\
\hline 25.405 & 378.3 & 320.5 & 333.4 & 3.5 & 6.4 \\
\hline 27.311 & 388.0 & 328.6 & 341.4 & 3.5 & 4.3 \\
\hline 21.726 & 343.4 & 289.5 & 301.3 & 4.2 & 34.3 \\
\hline 22.646 & 354.3 & 299.8 & 311.8 & 3.8 & 19.4 \\
\hline 23.312 & 360.3 & 304.6 & 317.2 & 3.8 & 14.4 \\
\hline 23.966 & 367.0 & 310.5 & 322.9 & 3.7 & 10.5 \\
\hline 24.965 & 371.5 & 314.8 & 327.6 & 3.6 & 8.7 \\
\hline 26.005 & 377.9 & 320.0 & 333.0 & 3.6 & 6.7 \\
\hline 28.884 & 391.5 & 332.1 & 345.0 & 3.3 & 3.9 \\
\hline 30.403 & 397.0 & 336.8 & 349.7 & 3.2 & 3.2 \\
\hline 25.965 & 378.5 & 320.6 & 333.5 & 3.5 & 6.4 \\
\hline 27.284 & 385.6 & 326.9 & 339.9 & 3.5 & 4.8 \\
\hline 27.338 & 385.6 & 327.0 & 340.0 & 3.4 & 4.8 \\
\hline 29.031 & 392.5 & 332.8 & 345.8 & 3.3 & 3.8 \\
\hline 30.537 & 397.6 & 337.0 & 350.2 & 3.2 & 3.2 \\
\hline 37.508 & 373.8 & 315.6 & 328.6 & 3.6 & 11.7 \\
\hline 38.721 & 379.5 & 320.8 & 334.2 & 3.5 & 9.2 \\
\hline 39.880 & 386.0 & 326.8 & 340.1 & 3.4 & 6.9 \\
\hline 41.706 & 392.9 & 332.4 & 345.9 & 3.4 & 5.3 \\
\hline 36.921 & 349.5 & 294.6 & 306.7 & 4.0 & 40.9 \\
\hline 37.734 & 355.3 & 299.6 & 311.7 & 3.8 & 30.5 \\
\hline 38.614 & 361.0 & 304.6 & 317.2 & 3.7 & 23.0 \\
\hline 38.614 & 361.0 & 304.5 & 317.2 & 3.7 & 23.0 \\
\hline 39.560 & 367.0 & 310.0 & 323.1 & 3.6 & 17.2 \\
\hline 40.693 & 373.5 & 315.6 & 328.6 & 3.5 & 12.8 \\
\hline 42.133 & 379.1 & 320.4 & 333.3 & 3.5 & 10.1 \\
\hline 44.159 & 387.4 & 327.6 & 341.1 & 3.4 & 7.2 \\
\hline 37.694 & 355.5 & 299.6 & 311.7 & 3.9 & 30.2 \\
\hline 38.587 & 361.3 & 304.9 & 317.4 & 3.7 & 22.7 \\
\hline 39.454 & 367.4 & 310.1 & 323.2 & 3.6 & 16.9 \\
\hline 40.760 & 374.4 & 316.4 & 329.3 & 3.5 & 12.4 \\
\hline 43.959 & 387.1 & 327.3 & 340.8 & 3.4 & 7.3 \\
\hline 47.771 & 398.4 & 337.0 & 350.7 & 3.3 & 4.8 \\
\hline 50.224 & 404.3 & 341.8 & 355.4 & 3.2 & 3.9 \\
\hline 52.796 & 409.5 & 346.3 & 360.0 & 3.1 & 3.3 \\
\hline 40.760 & 373.3 & 315.4 & 328.4 & 3.5 & 13.0 \\
\hline 41.946 & 379.4 & 320.4 & 333.3 & 3.5 & 10.0 \\
\hline 47.598 & 397.5 & 336.0 & 349.8 & 3.3 & 5.0 \\
\hline 47.691 & 397.9 & 336.0 & 349.8 & 3.4 & 4.9 \\
\hline 116.775 & 412.6 & 346.0 & 361.2 & 3.3 & 6.5 \\
\hline 121.214 & 418.5 & 351.4 & 366.6 & 3.2 & 5.3 \\
\hline 121.241 & 417.8 & 350.5 & 365.8 & 3.3 & 5.5 \\
\hline 126.172 & 423.6 & 355.9 & 370.7 & 3.1 & 4.6 \\
\hline 116.469 & 411.6 & 345.6 & 360.6 & 3.3 & 6.7 \\
\hline 121.027 & 417.3 & 350.5 & 365.6 & 3.2 & 5.6 \\
\hline 141.314 & 412.5 & 345.8 & 361.0 & 3.3 & 7.9 \\
\hline 145.713 & 417.8 & 350.5 & 365.8 & 3.2 & 6.6 \\
\hline 137.595 & 407.9 & 342.0 & 357.3 & 3.3 & 9.3 \\
\hline
\end{tabular}


TABLE III. Measured data for each experiment for trimethylene glycol. $T_{0}$, the temperature of the pool surface in Kelvin; $T_{1}$, the temperature of the top plate in Kelvin; $P_{t}$, the total pressure in $\mathrm{kPa} ; S_{\max }$, the maximum supersaturation in the chamber; $T_{\left(S_{\max }\right)}$, the temperature at $S_{\max }$, and and $P_{t} / P_{0}$ is the pressure ratio in the chamber where $P_{0}$ is the equilibrium vapor pressure of trimethylene glycol at $T_{0}$.

\begin{tabular}{|c|c|c|c|c|c|}
\hline$P_{\text {tot }}$ & $T_{0}$ & $T_{1}$ & $T\left(S_{\max }\right)$ & $S_{\max }$ & $P_{\mathrm{tot}} / P_{0}$ \\
\hline 22.873 & 363.5 & 295.0 & 307.2 & 6.8 & 29.1 \\
\hline 23.419 & 370.4 & 301.6 & 314.4 & 6.2 & 20.9 \\
\hline 23.952 & 375.9 & 306.3 & 319.6 & 6.0 & 16.3 \\
\hline 24.579 & 382.4 & 312.8 & 326.5 & 5.5 & 12.2 \\
\hline 26.045 & 394.8 & 322.0 & 335.8 & 5.6 & 7.3 \\
\hline 23.046 & 364.5 & 296.8 & 309.5 & 6.5 & 27.7 \\
\hline 23.486 & 370.8 & 302.0 & 314.7 & 6.2 & 20.5 \\
\hline 24.712 & 382.0 & 312.3 & 325.4 & 5.6 & 12.5 \\
\hline 27.111 & 399.3 & 327.1 & 340.8 & 5.1 & 6.3 \\
\hline 26.431 & 393.0 & 322.5 & 336.0 & 5.1 & 8.0 \\
\hline 27.351 & 399.0 & 328.5 & 342.5 & 4.7 & 6.4 \\
\hline 28.244 & 403.9 & 332.8 & 346.8 & 4.6 & 5.3 \\
\hline 29.364 & 409.1 & 337.5 & 351.8 & 4.4 & 4.4 \\
\hline 30.843 & 415.5 & 342.5 & 356.8 & 4.3 & 3.6 \\
\hline 32.736 & 421.8 & 348.5 & 362.7 & 4.0 & 3.0 \\
\hline 34.855 & 427.1 & 353.1 & 367.7 & 3.7 & 2.6 \\
\hline 25.898 & 382.0 & 315.0 & 328.3 & 4.8 & 13.1 \\
\hline 26.685 & 387.1 & 320.0 & 333.8 & 4.6 & 10.6 \\
\hline 27.604 & 395.0 & 326.1 & 339.9 & 4.6 & 7.7 \\
\hline 22.619 & 369.5 & 299.5 & 312.5 & 6.8 & 21.0 \\
\hline 23.979 & 375.5 & 304.9 & 317.8 & 6.4 & 16.5 \\
\hline 24.192 & 385.5 & 314.6 & 328.5 & 5.7 & 10.3 \\
\hline 24.899 & 390.6 & 319.6 & 333.3 & 5.4 & 8.4 \\
\hline 25.752 & 395.8 & 324.6 & 338.8 & 5.1 & 6.9 \\
\hline 26.618 & 400.6 & 328.6 & 342.9 & 5.0 & 5.8 \\
\hline 25.845 & 382.5 & 314.8 & 328.2 & 5.0 & 12.7 \\
\hline 26.738 & 387.9 & 320.0 & 333.8 & 4.8 & 10.3 \\
\hline 27.764 & 395.3 & 326.1 & 340.0 & 4.6 & 7.6 \\
\hline 28.871 & 401.9 & 331.3 & 345.3 & 4.6 & 5.9 \\
\hline 31.950 & 414.1 & 341.9 & 356.5 & 4.3 & 3.9 \\
\hline 33.722 & 420.3 & 346.6 & 361.4 & 4.1 & 3.3 \\
\hline 35.655 & 421.3 & 348.8 & 363.2 & 3.9 & 3.3 \\
\hline 36.575 & 423.5 & 350.8 & 365.5 & 3.8 & 3.1 \\
\hline 39.174 & 429.8 & 355.6 & 370.2 & 3.6 & 2.6 \\
\hline 51.010 & 390.0 & 321.1 & 335.1 & 4.6 & 17.8 \\
\hline 54.076 & 402.5 & 332.3 & 346.7 & 4.2 & 10.9 \\
\hline 48.784 & 384.3 & 315.8 & 329.9 & 4.8 & 22.1 \\
\hline 49.984 & 389.5 & 320.5 & 334.5 & 4.7 & 17.7 \\
\hline 53.183 & 403.6 & 332.5 & 347.1 & 4.4 & 10.1 \\
\hline 56.155 & 409.5 & 337.0 & 351.7 & 4.3 & 8.4 \\
\hline 56.795 & 415.5 & 341.9 & 356.6 & 4.3 & 6.7 \\
\hline 56.782 & 414.4 & 342.0 & 357.3 & 4.1 & 6.9 \\
\hline 58.728 & 419.8 & 346.5 & 361.9 & 4.0 & 5.8 \\
\hline 61.340 & 425.9 & 351.5 & 367.2 & 3.9 & 4.8 \\
\hline 64.352 & 432.0 & 356.4 & 371.9 & 3.9 & 4.0 \\
\hline 62.460 & 423.5 & 351.5 & 366.7 & 3.7 & 5.3 \\
\hline 50.850 & 396.3 & 321.5 & 335.8 & 5.6 & 13.4 \\
\hline 46.918 & 373.5 & 301.0 & 314.2 & 6.9 & 35.8 \\
\hline 47.718 & 377.8 & 305.5 & 319.2 & 6.4 & 29.5 \\
\hline 49.757 & 389.0 & 315.5 & 329.8 & 5.8 & 18.1 \\
\hline 50.943 & 394.9 & 320.1 & 334.4 & 5.7 & 14.2 \\
\hline 60.634 & 426.4 & 352.3 & 367.9 & 3.9 & 4.6 \\
\hline 39.534 & 379.5 & 310.5 & 324.0 & 5.3 & 22.5 \\
\hline 41.400 & 389.9 & 319.9 & 333.9 & 4.9 & 14.5 \\
\hline 44.252 & 405.3 & 333.1 & 347.7 & 4.6 & 7.9 \\
\hline 39.640 & 379.3 & 309.0 & 322.8 & 5.7 & 22.8 \\
\hline 42.933 & 398.3 & 328.0 & 342.4 & 4.5 & 10.3 \\
\hline 44.372 & 405.0 & 333.6 & 348.1 & 4.4 & 8.0 \\
\hline 49.437 & 394.3 & 323.9 & 337.9 & 4.7 & 14.2 \\
\hline 51.197 & 403.1 & 331.5 & 346.2 & 4.5 & 10.0 \\
\hline 52.596 & 410.3 & 338.0 & 352.5 & 4.3 & 7.6 \\
\hline 54.262 & 416.5 & 342.6 & 357.4 & 4.3 & 6.1 \\
\hline 55.502 & 420.5 & 346.1 & 361.0 & 4.2 & 5.3 \\
\hline 46.132 & 387.1 & 314.4 & 328.5 & 5.7 & 18.3 \\
\hline
\end{tabular}


TABLE IV. Measured data for each experiment for glycerol. $T_{0}$, the temperature of the pool surface in Kelvin; $T_{1}$, the temperature of the top plate in Kelvin; $P_{t}$, the total pressure in $\mathrm{kPa} ; S_{\max }$, the maximum supersaturation in the chamber; $T_{\left(S_{\max }\right)}$, the temperature at $S_{\max }$, and $P_{t} / P_{0}$ is the pressure ratio in the chamber where $P_{0}$ is the equilibrium vapor pressure of glycerol at $T_{0}$.

\begin{tabular}{|c|c|c|c|c|c|}
\hline$P_{\text {tot }}$ & $T_{0}$ & $T_{1}$ & $T\left(S_{\max }\right)$ & $S_{\max }$ & $P_{\mathrm{tot}} / P_{0}$ \\
\hline 8.477 & 415.1 & 329.0 & 340.8 & 24.5 & 24.3 \\
\hline 8.717 & 421.4 & 334.5 & 346.1 & 22.5 & 17.8 \\
\hline 7.984 & 390.3 & 307.8 & 318.1 & 35.4 & 99.6 \\
\hline 8.144 & 396.3 & 313.0 & 323.3 & 31.7 & 70.1 \\
\hline 8.317 & 401.8 & 317.8 & 328.9 & 28.8 & 51.4 \\
\hline 8.930 & 421.0 & 334.4 & 346.0 & 22.2 & 18.6 \\
\hline 9.210 & 426.0 & 338.5 & 350.9 & 21.1 & 14.7 \\
\hline 9.517 & 433.3 & 344.4 & 356.5 & 20.4 & 10.4 \\
\hline 9.437 & 430.9 & 342.0 & 354.4 & 21.1 & 11.7 \\
\hline 9.810 & 437.5 & 347.9 & 360.0 & 19.8 & 8.6 \\
\hline 10.637 & 449.5 & 357.0 & 370.0 & 18.9 & 5.3 \\
\hline 11.929 & 461.5 & 367.0 & 380.2 & 16.4 & 3.4 \\
\hline 12.889 & 468.9 & 372.0 & 385.1 & 15.2 & 2.6 \\
\hline 9.317 & 427.8 & 338.4 & 350.1 & 23.3 & 13.5 \\
\hline 10.423 & 445.5 & 353.8 & 366.7 & 19.4 & 6.2 \\
\hline 11.663 & 457.9 & 363.9 & 376.6 & 17.4 & 3.9 \\
\hline 8.637 & 399.4 & 313.9 & 324.4 & 34.5 & 61.6 \\
\hline 8.970 & 410.9 & 323.6 & 335.0 & 29.0 & 32.7 \\
\hline 9.224 & 417.0 & 328.9 & 340.1 & 26.8 & 23.8 \\
\hline 17.501 & 421.4 & 334.0 & 346.3 & 22.0 & 35.7 \\
\hline 18.381 & 433.6 & 343.5 & 355.8 & 20.2 & 19.7 \\
\hline 18.887 & 439.4 & 347.9 & 360.9 & 19.8 & 15.2 \\
\hline 19.474 & 446.5 & 353.3 & 366.2 & 19.4 & 11.1 \\
\hline 20.860 & 458.5 & 363.3 & 376.9 & 17.7 & 6.8 \\
\hline 18.967 & 439.6 & 348.8 & 361.7 & 19.0 & 15.1 \\
\hline 16.875 & 404.1 & 319.0 & 330.6 & 28.6 & 90.6 \\
\hline 17.194 & 410.0 & 323.9 & 335.4 & 26.4 & 66.0 \\
\hline 17.488 & 415.6 & 328.8 & 340.3 & 24.2 & 48.8 \\
\hline 18.661 & 432.3 & 342.8 & 355.0 & 20.0 & 21.5 \\
\hline 19.114 & 438.3 & 347.0 & 360.1 & 19.8 & 16.3 \\
\hline 16.341 & 391.0 & 308.6 & 319.1 & 33.8 & 193.9 \\
\hline 20.673 & 457.1 & 362.5 & 376.0 & 17.5 & 7.1 \\
\hline 19.247 & 439.0 & 347.6 & 360.7 & 19.7 & 15.7 \\
\hline 21.966 & 462.5 & 366.9 & 380.5 & 16.9 & 5.9 \\
\hline 24.019 & 474.5 & 376.9 & 390.3 & 14.9 & 3.9 \\
\hline 15.555 & 394.0 & 309.5 & 320.1 & 36.7 & 154.1 \\
\hline 15.728 & 399.5 & 314.4 & 325.0 & 32.7 & 111.6 \\
\hline 15.968 & 405.6 & 319.9 & 331.4 & 29.0 & 78.5 \\
\hline 16.715 & 416.6 & 328.5 & 340.2 & 25.8 & 44.1 \\
\hline 16.888 & 422.1 & 333.5 & 345.9 & 23.6 & 33.0 \\
\hline 17.248 & 427.9 & 337.5 & 350.0 & 23.2 & 24.9 \\
\hline 17.634 & 433.4 & 341.5 & 354.0 & 22.8 & 19.2 \\
\hline 15.288 & 392.5 & 309.4 & 319.9 & 34.3 & 166.2 \\
\hline 15.862 & 403.1 & 318.5 & 329.9 & 28.6 & 90.3 \\
\hline 16.528 & 415.5 & 328.5 & 340.0 & 24.6 & 46.6 \\
\hline 33.642 & 416.0 & 326.9 & 338.9 & 27.6 & 91.7 \\
\hline 34.336 & 423.0 & 333.0 & 345.1 & 24.4 & 64.0 \\
\hline 35.842 & 435.5 & 343.0 & 356.1 & 21.5 & 35.1 \\
\hline
\end{tabular}

this effect on the critical supersaturation of glycerol is shown in Fig. 6.

The effect of carrier gas pressure on the homogeneous nucleation of several compounds (apparent decrease in the nucleation rate with an increase in carrier gas pressure) was observed in DCC experiments reported by several groups. ${ }^{50-52}$ In those experiments the observed pressure effect increased with decreasing temperature or with increasing molecular weight of the condensing vapor. The present data on glycerol (Fig. 6) are consistent with the previously reported trends. It is interesting, however, to note that no effect of carrier gas pressure has been observed on the homogeneous nucleation rates measured in expansion cloud chambers ${ }^{7,53}$ or by Laval nozzle expansions. ${ }^{54}$

It is generally assumed that the role of the carrier gas in the nucleation process is only the thermalization of the embryonic droplets. In the limit of very low carrier gas pressures, the latent heat of condensation cannot be easily re- 
TABLE V. Thermophysical properties of ethylene glycol, propylene glycol, trimethylene glycol, and glycerol. Values of $M$, the molecular weight in $\mathrm{g} / \mathrm{mol} ; T_{c}$, the critical temperature in $\mathrm{K} ; P_{c}$, the critical pressure in $\mathrm{kPa}$; $T_{b}$, the normal boiling point in $\mathrm{K} ; D_{12}$, the binary diffusion coefficient at $273 \mathrm{~K}$, and $101.3 \mathrm{kPa} ; s$, the exponent of the temperature dependence; $a$, the thermal diffusion factor; $\Delta H_{\text {vap }}$, the enthalpy of vaporization at the normal boiling point in $\mathrm{cal} / \mathrm{mol} ; d$, the density of the liquid in $\mathrm{g} / \mathrm{cm}^{3} ; P_{e}$, the equilibrium vapor pressure in $\mathrm{kPa}$; $\sigma$, the surface tension in dyn/cm; $C_{p}$, the isobaric specific heat of the vapor in $\mathrm{J} / \mathrm{mol} \mathrm{K} ; \eta$, the viscosity of the vapor in poise; $\lambda$, the thermal conductivity of the vapor in $\mathrm{cal} / \mathrm{cm} \mathrm{s} \mathrm{K}$.

$$
\begin{aligned}
& \text { Ethylene glycol } \\
& M=62.069, T_{c}=645.0, P_{c}=7698, T_{b}=470.5 \\
& D_{12}^{\mathrm{a}}=0.34196, s^{\mathrm{a}}=0.75, a^{\mathrm{b}}=0.3, \Delta H_{\text {vap }}{ }^{\mathrm{c}}=14032.4 \\
& d^{\mathrm{d}}=1.1347-0.1026 E-2 * t-0.7094 E-6^{*} t^{2}\left(t: 7-116^{\circ} \mathrm{C}\right) \\
& \log P_{e}{ }^{\mathrm{e}}=9.394-3064.9 / T+\log (133.3)(T: 326-393 \mathrm{~K}) \\
& \sigma^{\mathrm{f}}=50.21-0.890^{*} t\left(t: 20-140^{\circ} \mathrm{C}\right) \\
& C_{p}{ }^{\mathrm{g}}=35.70+0.2483^{*} T-1.497 E-4 * T^{2}+3.010 E-8 T^{3} \\
& \eta^{\mathrm{h}}=0.9758 E-5 * T^{1.5} /(T+315.0)(T: 280-390 \mathrm{~K}) \\
& \lambda^{\mathrm{i}}=-0.1045 E-4+0.1144 E-6^{*} T+0.1333 E-9 * T^{2}(T: 280-390 \mathrm{~K}) \\
& \text { Propylene glycol } \\
& M=76.096, T_{c}=625.0, P_{c}=6060, T_{b}=460.5 \mathrm{~K} \\
& D_{12}{ }^{\mathrm{a}}=0.2924, s^{\mathrm{a}}=0.75, a^{\mathrm{b}}=0.3, \Delta H_{\text {vap }}{ }^{\mathrm{c}}=13575 \\
& d^{\mathrm{d}}=1.0609-1.1705 E-3 * t-1.0045 E-6 * t^{2}\left(t:-33-226^{\circ} \mathrm{C}\right) \\
& \log P_{e}{ }^{\mathrm{e}}=9.3300-2970.9 / T+\log (133.3)(T: 319-461 \mathrm{~K}) \\
& \sigma^{\mathrm{j}}=41.18-0.099^{*} t \mathrm{dyn} / \mathrm{cm}\left(t: 30-60{ }^{\circ} \mathrm{C}\right) \\
& C_{p f}=0.6322+0.4212 * T-2.981 E-4 * T^{2}+8.951 E-8 * T^{3} \\
& \eta^{\mathrm{h}}=0.9638 E-5^{*} T^{1.5} /(T+344.4)(T: 300-400 \mathrm{~K}) \\
& \lambda^{\mathrm{i}}=-1.0040 E-5+7.9335 E-8 * T+1.7004 E-10 * T^{2}(T: 240-400 \mathrm{~K}) \\
& \text { Trimethylene glycol } \\
& M=76.096, T_{c}=724.0, P_{c}=8901, T_{b}=487.6 \text {, } \\
& D_{12}{ }^{\mathrm{a}}=0.2924, s^{\mathrm{a}}=0.75, a^{\mathrm{b}}=0.3, \Delta H_{\text {vap }}{ }^{\mathrm{c}}=13782.3 \\
& d^{\mathrm{d}}=1.0775-0.8658 E-3 * t-0.5248 E-6 * t 2\left(t: 33-227^{\circ} \mathrm{C}\right) \\
& \log P_{e} e^{\mathrm{e}}=9.0479-3008.4 / T+\log (133.3)(T: 333-466 \mathrm{~K}) \\
& \sigma^{\mathrm{f}}=47.43-0.0903^{*} t\left(t: 20-140{ }^{\circ} \mathrm{C}\right) \\
& C_{p}{ }^{\mathrm{g}}=8.269+3.676 E-1 * T-2.162 E-4 * T^{2}+5.053 E-8 * T^{3} \\
& \eta^{\mathrm{k}}=0.9298 E-5^{*} T^{1.5} /(T+313.2)(T: 240-400 \mathrm{~K}) \\
& \lambda^{\mathrm{i}}=-0.1295 E-4+0.1328 E-6^{*} T+0.1497 E-9^{*} T_{2}(T: 240-400 \mathrm{~K}) \\
& \text { Glycerol } \\
& M=92.095, T_{c}=726.0, P_{c}=6678, T_{b}=563.0 \\
& D_{12}{ }^{\mathrm{a}}=0.2803 s^{\mathrm{a}}=0.75, a^{\mathrm{b}}=0.3, \Delta H_{\text {vap }}{ }^{\mathrm{c}}=18188.9 \\
& d^{\mathrm{d}}=1.2874-0.1293 E-2 * t-0.5837 E-6^{*} t^{2}\left(t:-33-227^{\circ} \mathrm{C}\right) \\
& \log P_{c}{ }^{\mathrm{e}}=10.4286-4155.9 / T+\log (133.3)(T: 400-563 \mathrm{~K}) \\
& \sigma^{\mathrm{l}}=65.55-0.9485^{*} t\left(t: 20-150{ }^{\circ} \mathrm{C}\right) \\
& C_{p}{ }^{\mathrm{g}}=8.424+4.442 E-1 * T-3.159 E-4 * T^{2}+9.378 E-8^{*} T^{3} \\
& \eta^{\mathrm{h}}=0.9879 E-5^{*} T^{1.5} /(T+378.2)(T: 300-500 \mathrm{~K}) \\
& \lambda^{\mathrm{i}}=-0.1490 E-4+0.1028 E-6^{*} T+0.1035 E-9^{*} T^{2}(T: 300-500 \mathrm{~K}) \\
& \text { Helium }^{\mathrm{m}} \\
& M=4.006 \\
& \eta=1.455 E-5 * T^{1.5} /(T+74.1)(T: 300-500 \mathrm{~K}) \\
& \lambda=7.37697 E-5+1.139222 E-6 * T+6.343536 E-10^{*} T^{2}(T: 270-400 \mathrm{~K})
\end{aligned}
$$

${ }^{\mathrm{a}}$ Reference 45 .

${ }^{\mathrm{b}}$ The thermal diffusion factor is approximated as $a=0.3$ by analogy to the values for other mixtures found in $\mathrm{K}$. E. Grew and T. L. Ibbs, Thermal Diffusion of Gases (Cambridge University Press, Cambridge, 1952), pp. 128-130.

${ }^{c}$ Reference 46, p. C727.

${ }^{\mathrm{d}}$ Reference 47, Eq. (3-11.11), p. 67.

${ }^{\mathrm{e}}$ Reference 46, p. D204.

${ }^{\mathrm{f}}$ Reference 48.

${ }^{g}$ Reference 47.

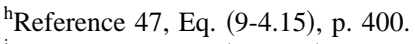

${ }^{i}$ Reference 47, Eq. (10-3.17), p. 505.

${ }^{j}$ Surface tension measurements were carried out at Philip Morris Research Center, Richmond, VA (Dr. Peter Lipowicz, unpublished data).

${ }^{\mathrm{k}}$ Reference 47, Eq. (9-4.9), p. 396.

${ }^{\mathrm{l}}$ Reference 46, p. F37.

${ }^{\mathrm{m}}$ Reference 49 . 


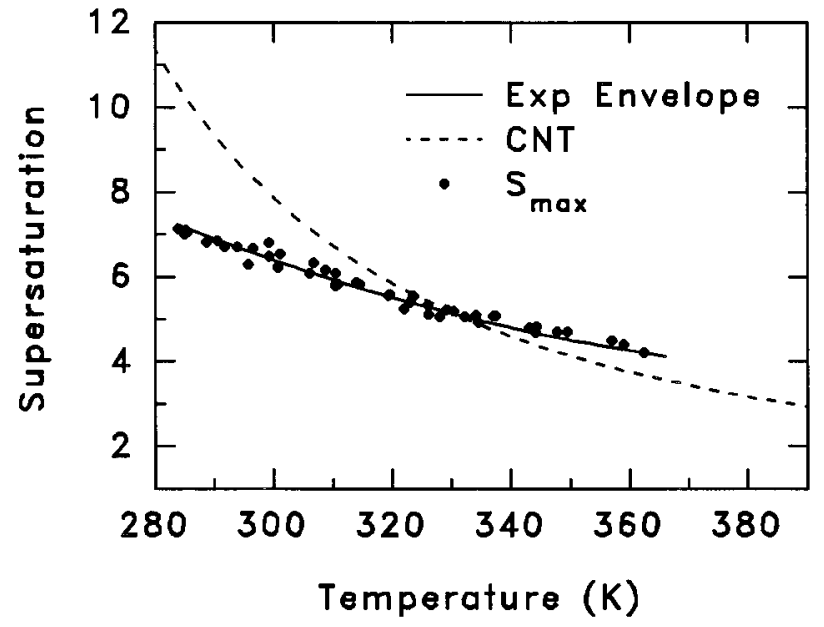

FIG. 2. Critical supersaturation vs temperature for ethylene glycol. Dashed line represents theory. Solid curve represents the experimental envelope of critical supersaturation, and dots represent the maximum supersaturations achieved in the chamber.

moved from newly formed clusters and this should lead to higher critical supersaturations. At intermediate pressure range (typically in the range of several Torr), the removal of the heat of condensation is effective and further increase in the carrier gas pressure should have no effect on the thermalization of the clusters. In fact, theoretical calculations suggest that the thermalization effect of the carrier gas is unmeasurably small in typical homogeneous nucleation experiments. ${ }^{55,56}$ Furthermore, by treating the nucleation process in the presence of a carrier gas as a binary system, Oxtoby and Laaksonen have arrived at the conclusion that the effect of the carrier gas pressure on both the rate and the critical supersaturation is very small. ${ }^{57}$ In addition, recent

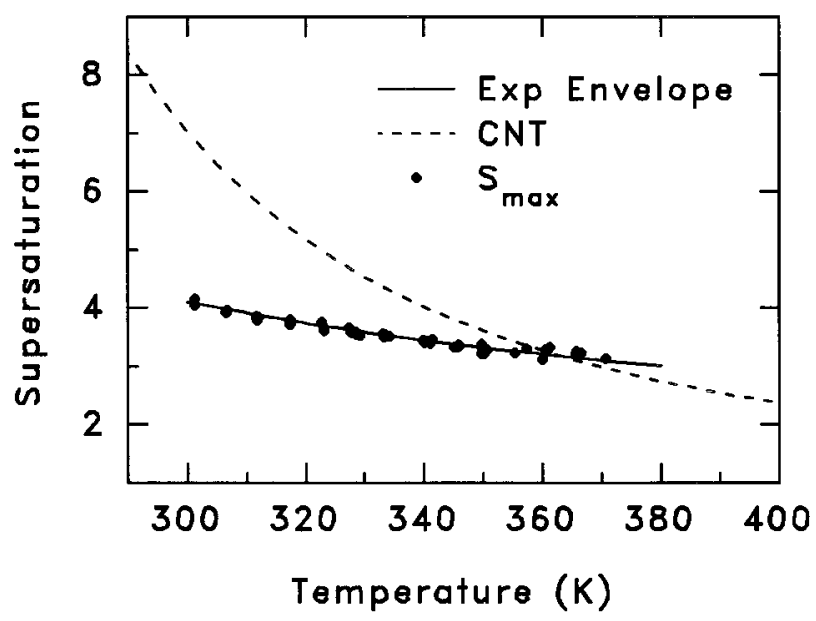

FIG. 3. Critical supersaturation vs temperature for propylene glycol. Dashed line represents theory. Solid curve represents the experimental envelope of critical supersaturation, and dots represent the maximum supersaturations achieved in the chamber.

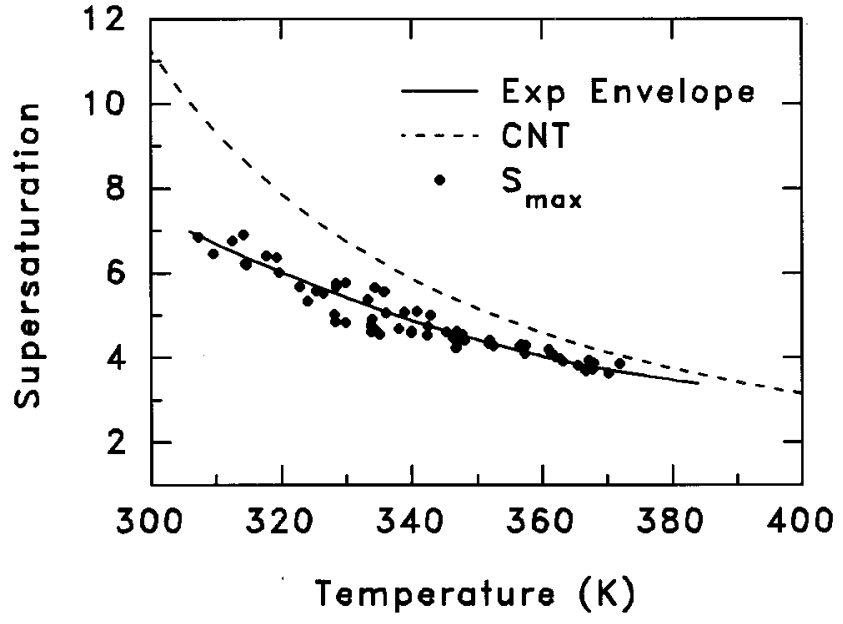

FIG. 4. Critical supersaturation vs temperature for trimethylene glycol. Dashed line represents theory. Solid curve represents the experimental envelope of critical supersaturation, and dots represent the maximum supersaturations achieved in the chamber.

analysis by Kashchiev has confirmed the conclusion of Oxtoby and Laaksonen by showing that the effect of the carrier gas is relatively small when the total pressure is not much greater than the atmospheric pressure. ${ }^{58}$

Considering that significant pressure effects on the nucleation process have been observed in the DCC experiments which could not be explained by nonideality and Poynting effects, ${ }^{59}$ one must consider the effect of pressure on the growth of the nuclei into macroscopic droplets which are usually detected by light scattering in order to count the nucleation events. We have investigated this effect by solving the equations of motion and growth of the droplets within a DCC and the details of the calculations are reported

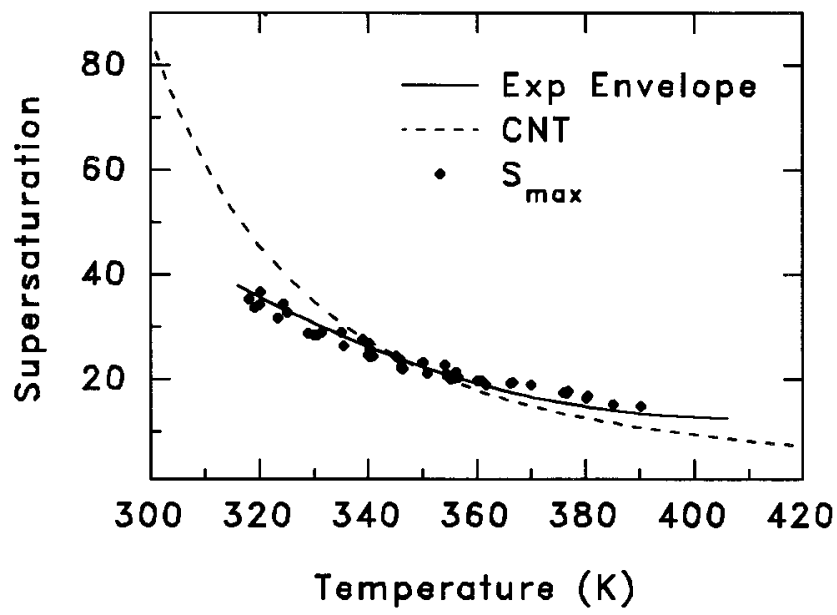

FIG. 5. Critical supersaturation vs temperature for glycerol. Dashed line represents theory. Solid curve represents the experimental envelope of critical supersaturation, and dots represent the maximum supersaturations achieved in the chamber. 


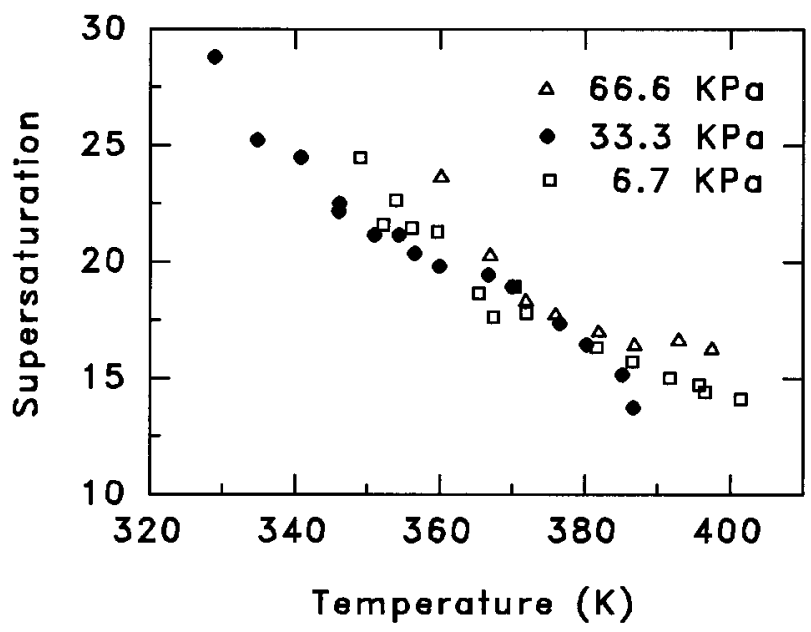

FIG. 6. Effect of carrier gas pressure (helium) on the critical supersaturation of glycerol.

elsewhere. ${ }^{60}$ Here, we only present the main conclusions for the effect of total pressure on the time required for the growth of nuclei into detectable droplets.

It can be shown that increasing the carrier gas pressure slows down the rate of growth of the droplets within the high supersaturation region of the cloud chamber thus the droplets fall (due to gravity effects) before they have enough time to become detectable objects. Since the intensity of light scattered by the droplet is proportional to $R^{2}$ (where $R$ is the radius of the droplet), it follows that at higher pressures droplets may not grow large enough that significant amounts of light are scattered. This could lead to an underestimation of the nucleation rates at higher pressures. An interesting possibility could arise if DCC nucleation experiments are conducted in a microgravity environment where the effect of gravity can be fully accounted for or eliminated. This will make it possible to increase the total pressure in the DCC while keeping the droplets within the region of maximum supersaturation for longer times. In this way, one can accurately examine the effect of increasing carrier gas pressure on the rate of nucleation.

\section{A. Comparison with the classical theory}

The prediction of the CNT for the dependence of $S_{c}$ on $T$ is computed from

$$
J=(a / d)\left(2 N_{A}^{3} \sigma M / p\right)^{1 / 2}\left(S P_{e} / R T\right)^{2} \exp \left(-W^{*} / R T\right),
$$

where, $J$ is the rate of nucleation $\left(\mathrm{cm}^{-3} \mathrm{~s}^{-1}\right), a$ is a sticking coefficient and is set equal to $1, d$ is the liquid density, $N_{A}$ is Avogadro's number, $\sigma$ is the flat surface tension of the liquid, $M$ is the molecular weight, $S$ is the supersaturation ratio $\left(P / P_{e}\right)$ where $P$ is the pressure of the vapor, and $P_{e}$ is the equilibrium or "saturation" vapor pressure at the temperature of the vapor $T$, and $R$ is the gas constant. The central quantity in the rate equation is the barrier height $W^{*}$ which is given by
TABLE VI. Comparison between experimental, $S$ (exp) and calculated, $S$ (CNT) supersaturations at different temperatures.

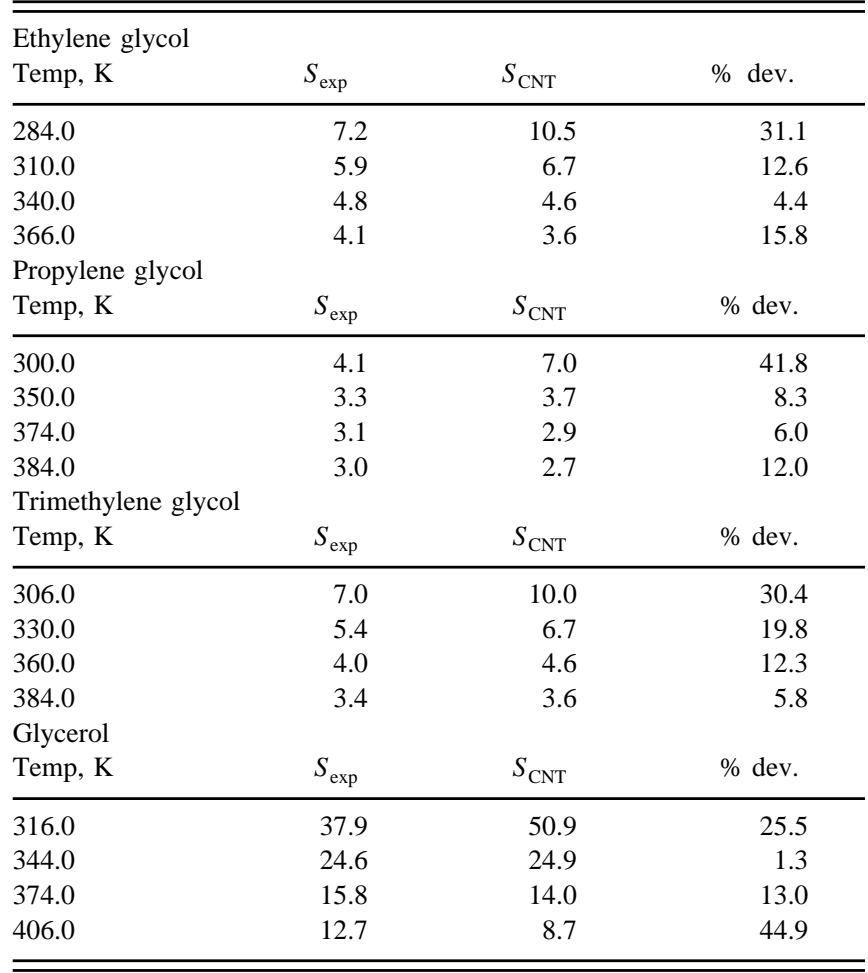

$$
W^{*}=16 \pi N_{A} M^{2} \sigma^{3} / 3(d R T \ln S)^{2} .
$$

By setting $J=1$ in Eq. (1) and using the literature values of the equilibrium vapor pressure, liquid density and surface tension (as listed in Tables V), the dependence of $S_{c}$ on $T$ is obtained for each compound. The results are shown as the dashed curves in Figs. 2-5.

It is clear from the results in Figs. $2-5$ that the predictions of the CNT deviate strongly from the experimental results at lower temperatures. The magnitude of the discrepancy in $S_{c}$ is shown in Table VI where we compare the experimental and theoretical $S_{c}$ for all the investigated compounds at four different temperatures within the range of the experimental measurements.

It should also be noted that critical supersaturations measured for other hydrogen bonding substances such as methanol, ethanol, and propanol show changes in the slope of the temperature dependence of the critical supersaturation different from the predictions of the classical theory with a crossover at some temperature which depends on the molecular size of the alcohol. ${ }^{61-63}$ The present data for glycols and glycerol is consistent with that general trend previously observed although the magnitude and the sign of the temperature dependence effect are different from alcohol data.

Since the CNT predicts temperature dependences of $S_{c}$ for these systems which are significantly different from the experimental results, this raises the question of how the surface tension of the critical cluster compares to the bulk value. A significant decrease in the bulk surface tension at lower temperatures would be required to bring the CNT into agreement with the experimental results. This suggests that if the 


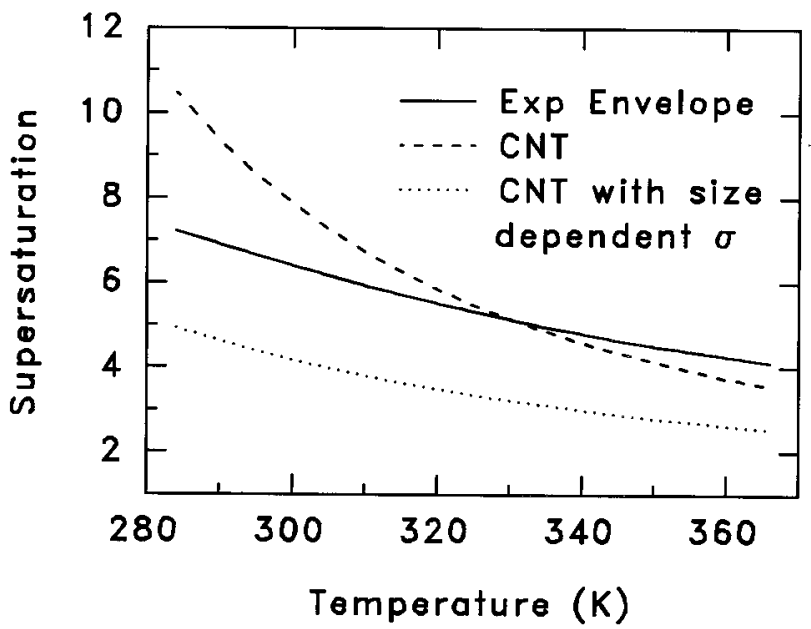

FIG. 7. Critical supersaturation vs temperature calculated using size dependent surface tension for ethylene glycol (dotted line). Solid curve represents the experimental results and dashed curve the CNT results (with bulk surface tension).

CNT is essentially correct, the embryonic droplets of the glycol molecules might be adequately described by smaller surface tensions than their bulk values. Note that at lower temperatures the critical nuclei are smaller. Therefore, the present results suggest that the surface tension of the critical clusters of glycols and glycerol could be considerably smaller than bulk values. This effect has been observed in the molecular dynamics simulations of small clusters. Thompson et al. have reported surface tensions which increase with size for clusters of 54 to 896 Lennard-Jones particles. ${ }^{64}$ Shreve et al. have reported similar results for clusters of 50 to 896 Stockmayer molecules. ${ }^{65}$ Also, Georgy

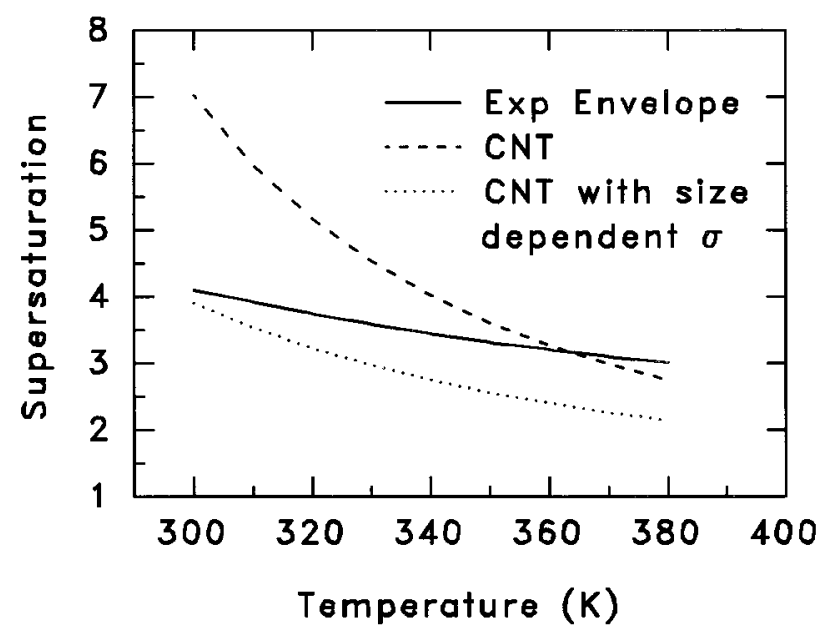

FIG. 8. Critical supersaturation vs temperature calculated using size dependent surface tension for propylene glycol (dotted line). Solid curve represents the experimental results and dashed curve the CNT results (with bulk surface tension).

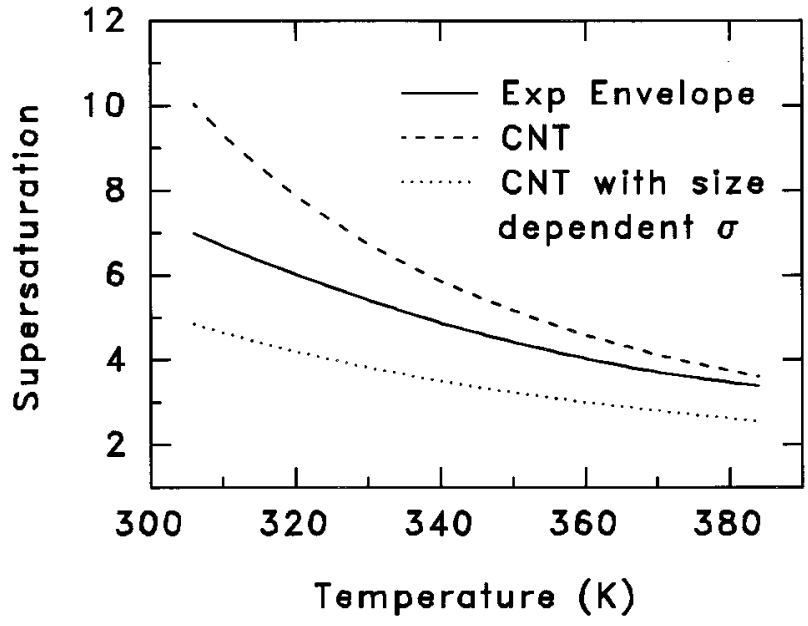

FIG. 9. Critical supersaturation vs temperature calculated using size dependent surface tension for trimethylene glycol (dotted line). Solid curve represents the experimental results and dashed curve the CNT results (with bulk surface tension).

and Schug have found that a size dependent surface tension was required to bring cluster distributions predicted by the classical nucleation theory into agreement with the results of their Monte Carlo simulations of Lennard-Jones particles. ${ }^{66}$

The dependence of droplet surface tension on curvature was examined using thermodynamic arguments by Tolman ${ }^{67}$ and statistical mechanical theory by Kirkwood and Buff. ${ }^{68}$ Based on their work, Abraham presented a simplified " order of magnitude" argument for the curvature dependence surface tension which can be written as ${ }^{69}$

$$
\sigma(r)=\sigma(\infty) /(1+2 \delta / r),
$$

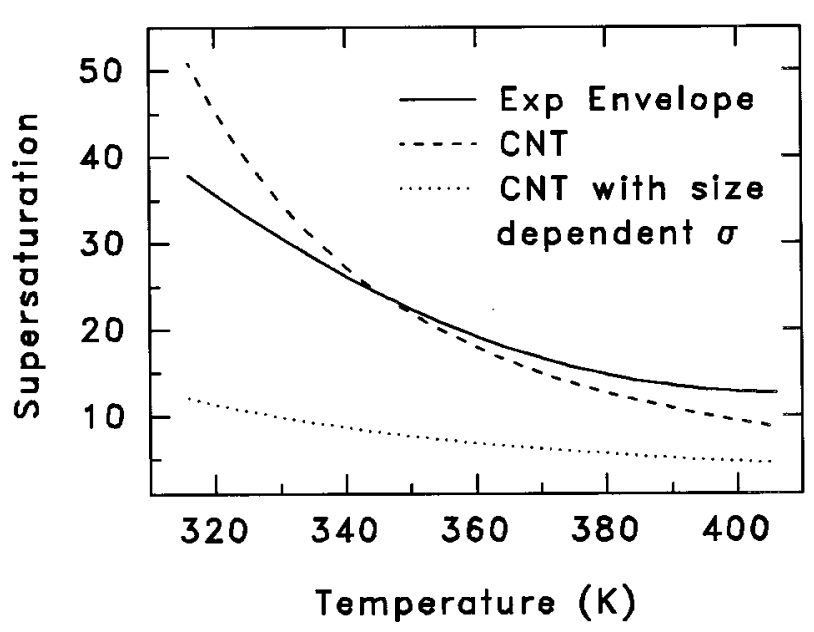

FIG. 10. Critical supersaturation vs temperature calculated using size dependent surface tension for glycerol (dotted line). Solid curve represents the experimental results and dashed curve the CNT results (with bulk surface tension). 


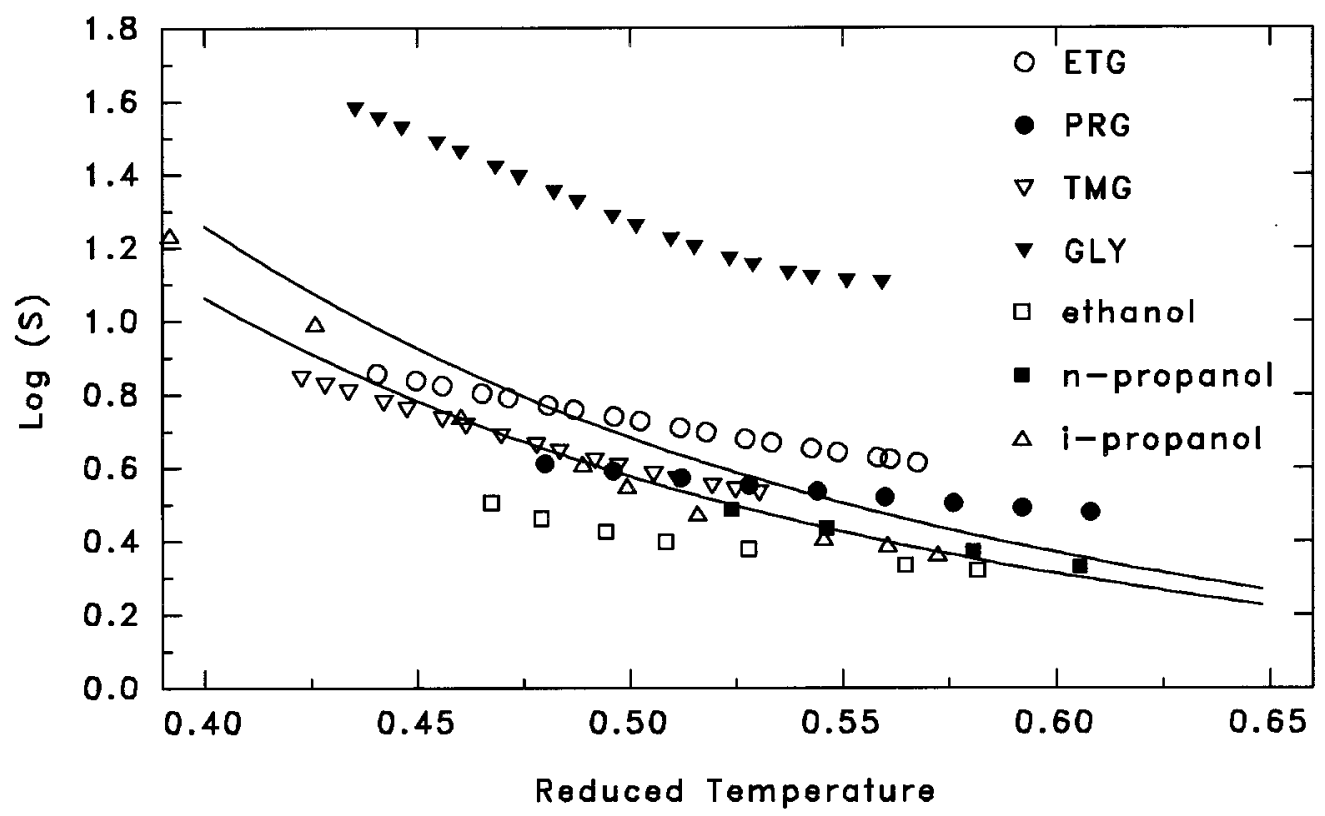

FIG. 11. Critical supersaturation vs reduced temperature. The lower and upper solid curves are for simple fluids and represent barrier heights of 70 and $50 \mathrm{kT}$, respectively. ETG, PRG, TMG, and GLY denote ethylene glycol, propylene glycol, trimethylene glycol, and glycerol, respectively.

where, $\sigma(r)$ is the surface tension of a droplet of radius $r$, $\sigma(\infty)$ is the surface tension at zero curvature $(r=\infty)$, and $\delta$ is half the thickness of a molecular layer in the liquid. It should be pointed out, however, that the application of Eq. (3) to very small critical clusters may only be valid when $2 \delta / r \ll 1$.

In order to investigate the effect of size-dependent surface tension, we used the following correction to the curvature dependent surface tension: ${ }^{70}$

$$
\sigma\left(r^{*}, T\right)=\sigma_{T}\left(1-r_{1} / r^{*}\right),
$$

where $r_{1}$ is the radius of the monomer and $r^{*}$ is the radius of the critical cluster. Using this equation we obtained new expressions for the barrier height and the rate of nucleation. The critical supersaturations obtained using the curvature dependent surface tensions are shown in Figs. 7-10. It is clear from these figures that using the curvature dependent surface tension specified by Eq. (4) results in supersaturations much less than the experimental results. Of course, it is possible to consider $r_{1}$ in Eq. (4) as an adjustable parameter in order to obtain the best agreement with the experimental results. It is interesting to note that at lower temperatures the surface tensions of the critical clusters required to reproduce the experimental supersaturations are smaller than the bulk values. For glycerol, ethylene glycol, and propylene glycol, the experimental and theoretical curves of $S_{c}$ vs $T$ cross each other at reduced temperatures corresponding to $0.48,0.51$, and 0.58 , respectively. At these temperatures the corresponding critical nuclei contain 32, 69, and 103 molecules, respectively. We note that the cross over point occurs at lower $T_{r}$ for glycerol than for the other compounds. This may be related to the extent of hydrogen bonding which is expected to be stronger in glycerol since the molecule contains three available hydrogen bonding sites. If such a correlation exists, it would appear that increasing the extent of hydrogen bonding in the clusters may result in smaller critical clusters which can be described by a smaller surface tension parameter compared to the corresponding bulk value. Therefore, the observed

TABLE VII. Molecular properties and critical supersaturations of some hydrogen bonding compounds.

\begin{tabular}{lcccccc}
\hline \hline \multicolumn{1}{c}{ Compound } & $T_{r}{ }^{\mathrm{a}}$ & $T$ & $\omega^{\mathrm{b}}$ & $\mu^{\mathrm{c}}$ & $S_{\exp }$ & $S_{\mathrm{CNT}}$ \\
\hline Ethylene glycol & 0.52 & 335.4 & 1.15 & 2.2 & 4.94 & 5.17 \\
Propylene glycol & 0.52 & 325.0 & 1.12 & 3.6 & 3.66 & 5.17 \\
Trimethylene glycol & 0.52 & 376.5 & 0.70 & 3.7 & 3.34 & 4.07 \\
Glycerol & 0.52 & 377.5 & 1.51 & 3.0 & 15.2 & 15.7 \\
Ethanol & 0.52 & 268.3 & 0.64 & 1.7 & 2.43 & 2.36 \\
Propanol & 0.52 & 279.2 & 0.62 & 1.7 & 2.99 & 2.91 \\
Isopropanol & 0.52 & 264.2 & 0.67 & 1.7 & 3.07 & 3.00 \\
\hline \hline
\end{tabular}

${ }^{\mathrm{a}} T_{r}$ is the reduced temperature $\left(T / T_{c}\right)$.

${ }^{\mathrm{b}} \omega$ is the Pitzer acentric parameter calculated from Ref. 47, Eq. (2-3.1).

${ }^{c} \mu$ is the dipole moment in debye taken from Ref. 47 . 
TABLE VIII. Calculated and predicted (from the experimental critical supersaturations) values of $\Omega$.

\begin{tabular}{lcccc}
\hline \hline \multicolumn{1}{c}{ Compound } & Temp, $\mathrm{K}$ & $\Omega_{1}$ & $\Omega_{2}$ & $\Omega^{\mathrm{a}}$ \\
\hline Ethylene glycol & 325 & 2.16 & 1.35 & 1.25 \\
Propylene glycol & 340 & 2.25 & 1.83 & 0.97 \\
Trimethylene glycol & 345 & 1.95 & 1.63 & 1.05 \\
Glycerol & 355 & 2.89 & 1.76 & 2.02 \\
\hline \hline
\end{tabular}

${ }^{\mathrm{a} B a s e d}$ on the experimental data shown in Fig. 16.

cross over between the experimental and theoretical curves of $S_{c}$ vs $T$ could be interpreted as a surface tension transition from bulk to cluster values. It should be mentioned that a similar behavior has been observed in the homogeneous nucleation of mercury which also nucleates at much lower supersaturations than is expected from the CNT. That behavior could be explained by noting that small clusters of mercury are distinctly nonmetallic, so that their effective surface free energy will be very different from that of metallic bulk mercury. ${ }^{17}$

\section{B. Corresponding states correlation}

Because of the approximate nature of the CNT, corresponding states and scaling laws are necessary in order to correlate trends in critical supersaturations with molecular properties. The sensitivity of vapor-to-liquid nucleation to small changes in thermodynamic properties suggests that nucleation of a simple fluid, all other things being equal, should be markedly different from the nucleation of a slightly different structured fluid. This can only be seen when the nucleation thresholds are expressed in a suitable dimensionless, corresponding states form. In this case, one can examine possible correlations between molecular properties and the critical supersaturations required to cause a vapor to condense. The nucleation behavior of simple fluids

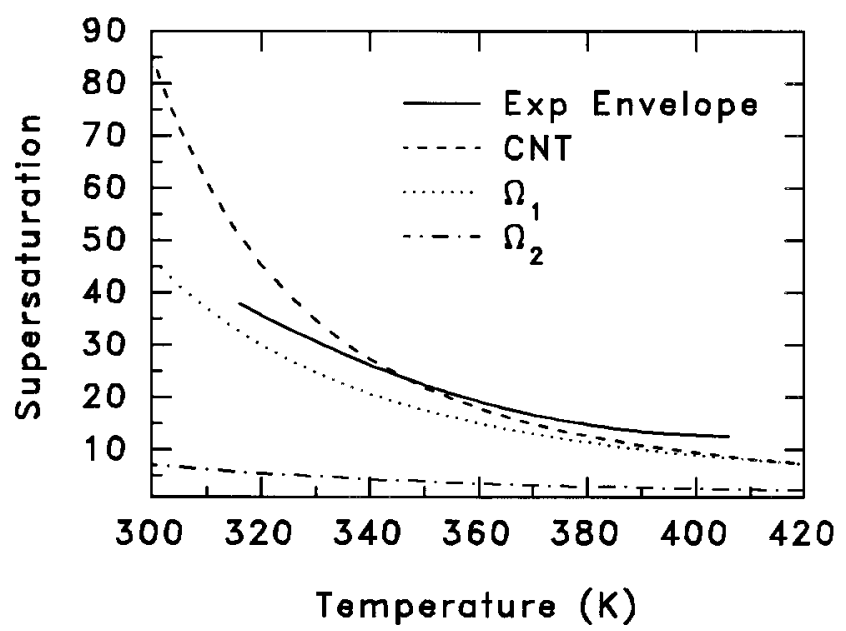

FIG. 12. Critical supersaturation vs temperature as predicted from the scaling law using $\Omega_{1}$ and $\Omega_{2}$ for ethylene glycol.

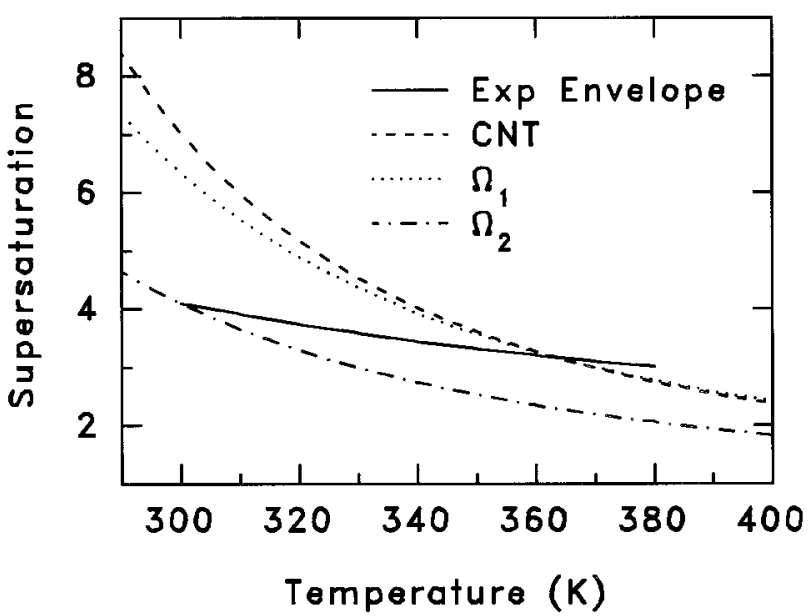

FIG. 13. Critical supersaturation vs temperature as predicted from the scaling law using $\Omega_{1}$ and $\Omega_{2}$ for propylene glycol.

serves as a reference point from which the systematic deviations due to complex fluids can be revealed and easily associated with a particular molecular property.

$\mathrm{McGraw}^{71}$ derived the following dimensionless form for the free energy maximum of nucleation, $W^{*}$,

$$
W^{*} / k_{B} T=282.3 G\left(T_{r}\right)(\log S)^{-2},
$$

where

$$
G\left(T_{r}\right)=\frac{T_{r}^{-3}\left(1-T_{r}\right)^{11 / 3}}{\left[1+0.75\left(1-T_{r}\right)+1.75\left(1-T_{r}\right)^{1 / 3}\right]^{2}},
$$

$k_{B}$ is Boltzmann's constant, and $T_{r}=T / T_{c}$. Equation (5) is based on Guggenheim's empirical correlations of the surface tension and the number density of the heavier rare gases and therefore is a good approximation for the reduced barrier height of simple fluids. For nucleation rates near unity $(J \sim 1$

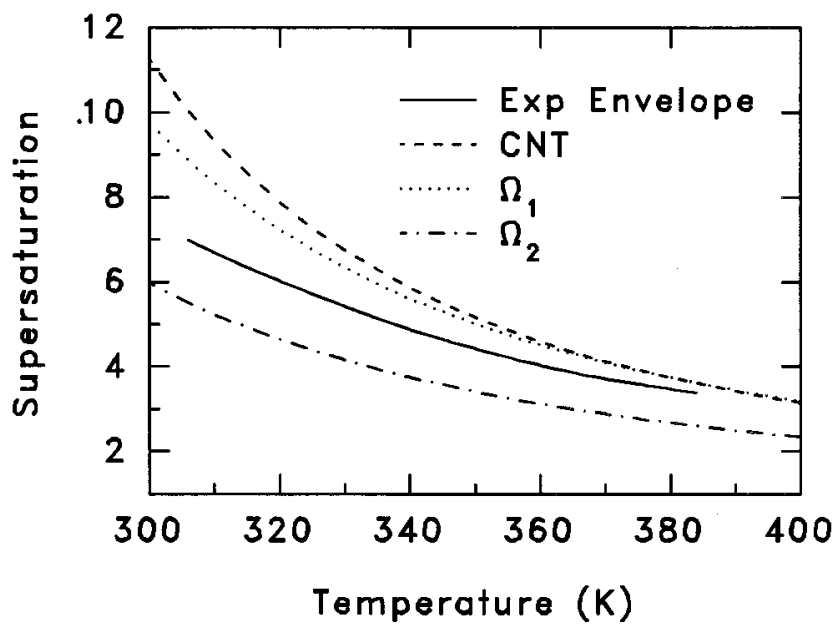

FIG. 14. Critical supersaturation vs temperature as predicted from the scaling law using $\Omega_{1}$ and $\Omega_{2}$ for trimethylene glycol. 
$\left.\mathrm{cm}^{-3} \mathrm{~s}^{-1}\right) W^{*}$ lies between 50 and $70 k_{B} T$. The dependence of supersaturation on $T_{r}$ for simple fluids is shown in Fig. 10 as two solid curves for barrier heights of 50 and $70 k_{B} T$ along with the experimental $S_{c}$ values for the studied compounds. It is interesting to note that the glycols (with the exception of glycerol) fall within the curves corresponding to simple fluid nucleation behavior. Similar observations have been found for other fluids such as $\mathrm{NH}_{3}, \mathrm{CHCl}_{3}$, and $\mathrm{CCl}_{3} \mathrm{~F}$ and for associated vapors such as acetic and formic acids. McGraw pointed out that the corresponding states of the nucleation thresholds incorporates the effect of vapor association in an approximate way. This is because $\epsilon_{0} / k_{B} T_{c}$ is a universal constant for simple fluids and hence any increase in $\epsilon_{0}$ due to association must be accompanied by an increase in $T_{c}$ to keep $\epsilon_{0} / k_{B} T_{c}$ constant. This leads to a reduction in $T_{r}$ which would mean an increase in $S_{c}$ of the associated vapors to conform to the pattern of simple fluids.

Figure 11 also compares the nucleation behavior of the glycols with the monohydroxy compounds ethanol, $n$-propanol, and isopropanol. It is clear that the critical supersaturation increases with increasing size of the alcohol. It is interesting to note that trimethylene glycol, propylene glycol, $n$-propanol, and isopropanol lie almost on the same curve.

Table VII lists the Pitzer acentric parameters, dipole moments, and critical supersaturations at $T_{r}=0.52$ for the compounds shown in Fig. 10. McGraw observed an increase in the critical supersaturation at a given reduced temperature with increasing the Pitzer acentric parameter, $\omega$ among a series of structurally related molecules. ${ }^{71}$ This trend is evident in the experimental data shown in Fig. 10. Glycerol which has the largest value of $\omega$ also exhibits the highest critical supersaturation and the smallest nucleus size.

The corresponding states results suggest different nucleation behavior for partially hydroxylated compounds than for completely hydroxylated compounds. The partially hydroxylated propylene and trimethylene glycols behave similarly to the corresponding alcohols, but ethylene glycol and glycerol are completely different. In these molecules the interaction is mainly $\mathrm{H}$ bonding and all the carbon sites carry $\mathrm{OH}$ groups. Within the critical clusters of propylene and trimethylene glycols the molecules interact via $\mathrm{H}$ bonds as well as by hydrophobic interaction.

The most prominent feature in Fig. 11 is the unique position of glycerol in comparison to the other glycols. The higher critical supersaturation of glycerol leads to quite small critical clusters $(20-50$ molecules at $300-400 \mathrm{~K})$. This is consistent with the known tendency of glycerol to form more stable aerosols compared to the other glycols.

\section{Scaled nucleation model}

In this section, we analyze our results in terms of the scaled nucleation model developed by Hale. ${ }^{72-75}$ For a nucleation rate of $1 \mathrm{~cm}^{-3} \mathrm{~s}^{-1}$, the scaling law is expressed as

$$
\ln S_{c}=0.53 \Omega^{3 / 2}\left[\left(T_{c} / T\right)-1\right]^{3 / 2},
$$

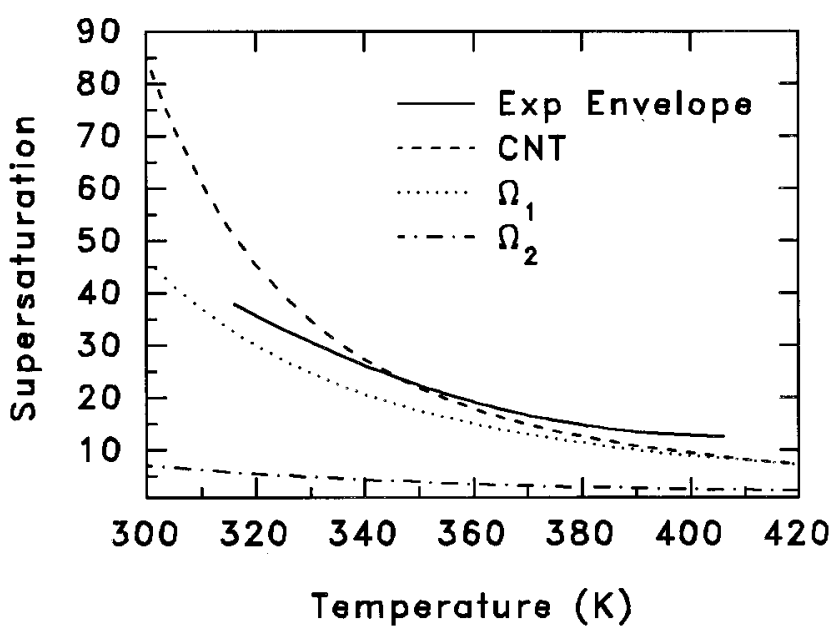

FIG. 15. Critical supersaturation vs temperature as predicted from the scaling law using $\Omega_{1}$ and $\Omega_{2}$ for glycerol.

where $\Omega$ is the excess surface entropy per molecule in the cluster and can be approximated by the Eotvos constant, $K_{e}$, which is defined as

$$
k_{B} N_{A}^{2 / 3} \Omega \approx K_{e}=v^{2 / 3} \sigma /\left(T_{c}-T\right) .
$$

Hale pointed out that a more reliable value for $\Omega$ can be obtained from the temperature dependence of the surface tension according to

$$
\Omega=-\left[\frac{\partial \sigma}{\partial T}\right] /\left(k_{B} \rho^{2 / 3}\right),
$$

where $\rho$ is the liquid number density. In order to investigate the effect of the parameter $\Omega$ on the predictive ability of the scaling law, we used Eqs. (8) and (9) to calculate $\Omega$ for the studied compounds at a midrange temperature of our data. The results are given in Table VIII where $\Omega$ calculated from Eqs. (8) and (9) are referred to as $\Omega_{1}$ and $\Omega_{2}$, respectively. Using these parameters, we calculated the critical supersaturation as a function of temperature using Eq. (7). The results as shown in Figs. 12-15, indicate that the choice of $\Omega$ has a crucial effect on the calculated supersaturation. It is also evident that $\Omega_{1}$ calculated from Eq. (8) does a relatively better job in predicting the critical supersaturation than $\Omega_{2}$ does, with the exception of propylene glycol where $\Omega_{2}$ appears to give supersaturations closer to the experimental values.

The measured supersaturations can be used to extract values for $\Omega$ from Eq. (7). Figure 15 exhibits plots of $\ln S_{c}$ vs $\left[\left(T_{c} / T\right)-1\right]^{3 / 2}$ based on the experimental values of $S_{c}$. The linearities of the plots are evident and from the slopes of the lines we calculate the $\Omega$ values which are listed also in Table VIII.

The most prominent feature in Fig. 16 is the grouping of the compounds in a similar fashion to that found with the corresponding states of simple fluids discussed in Sec. III B. Again, glycerol shows the highest supersaturation followed 


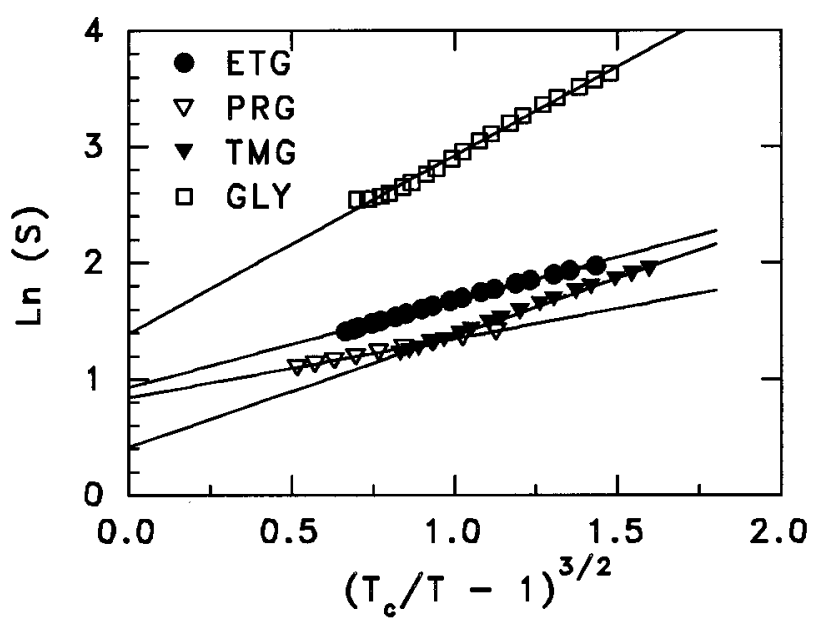

FIG. 16. ( $\left.\ln S_{c}\right)$ vs $\left[T_{c} / T-1\right]^{3 / 2}$ for the studied compounds. ETG, PRG, TMG, and GLY denote ethylene glycol, propylene glycol, trimethylene glycol, and glycerol, respectively.

by ethylene glycol. Therefore the characteristic nucleation behavior of the glycerol is clearly seen from the two scaled models.

\section{CONCLUSIONS}

(1) The critical supersaturations required for the homogeneous nucleation (rate of $1 \mathrm{drop} / \mathrm{cm}^{3} / \mathrm{s}$ ) of ethylene glycol, propylene glycol, trimethylene glycol and glycerol vapors have been measured over wide temperature ranges (e.g., 280-400 K) using an upward thermal diffusion cloud chamber. At lower temperatures the experimental nucleation rates are much higher than the predictions of the classical nucleation theory. Glycerol shows the best agreement between experiment and theory in the temperature range of 340-370 $\mathrm{K}$. However, at lower temperatures (300-330 K) the measured critical supersaturations are lower than the predictions of the theory, which is the case with all the studied glycols.

(2) A small apparent increase in the critical supersaturation with carrier gas pressure has been observed in the diffusion cloud chamber. This effect is more pronounced at lower temperatures and with increasing molecular weight of the condensing vapor. It is suggested that the pressure effect arises from the dynamics of growth and motion of the droplets and not from a pressure dependence of the kinetics of homogeneous nucleation. ${ }^{60}$

(3) The results from corresponding states and scaled nucleation models indicate that the nucleation behavior of glycerol is quite different from other glycols. Glycerol requires higher critical supersaturations compared to the other glycols at the same reduced temperatures. This leads to quite small critical nuclei for glycerol (20-50 molecules). This result explains the well known tendency of glycerol to form stable aerosols. This behavior could also be related to the formation of strong hydrogen-bonding networks within the liquid droplets of glycerol.
(4) The discrepancy between experiment and theory at lower temperatures may be explained by considering that the surface tensions of the critical clusters below certain temperatures are smaller than the bulk surface tensions. Further theoretical work is required in order to fully understand the observed phenomenon of higher nucleation rates of glycols and glycerol at lower temperatures. We are currently investigating the applications of other modified forms of the classical nucleation theory to hydrogen bonding substances such as glycols and glycerol. ${ }^{32,76-78}$ Future work will also involve Monte Carlo simulations of the molecular clusters of these systems. ${ }^{79,80}$

(5) To extend the range of operation of the diffusion cloud chamber for homogeneous nucleation measurements and for other applications including gas phase polymerization and nanocomposite materials, it is necessary to eliminate convection, wall effects and other instability factors. The microgravity environment appears to provide a unique opportunity in this regard. It is suggested to carry out homogeneous nucleation measurements in a diffusion cloud chamber within a high quality, long duration, microgravity environment. These measurements, in the absence of convection and gravity effects, would provide critical tests to the effect of carrier gas pressure which has been a source of controversy and thus would allow complete evaluation of the various theories of nucleation and growth. The microgravity environment would also provide new applications for the cloud chambers particularly in the control and design of novel composite materials produced by nucleation from the vapor phase.

\section{ACKNOWLEDGMENTS}

This work was supported by a grant from Philip Morris USA. We thank Dr. Peter Lipowicz for the surface tension measurements of propylene glycol and Dr. Cliff Lilly for his support and encouragement. Acknowledgment is made to NASA Microgravity Materials Science Program (NAG81276), to the donors of the Petroleum Research Fund (30630-AC6), administered by the American Chemical Society and to the Thomas F. and Kate Miller Jeffress Memorial Trust for the partial support of this research.

${ }^{1}$ Nucleation and Atmospheric Aerosols, edited by N. Fukuta and P. E. Wagner (Deepak, Hampton, 1992).

${ }^{2}$ Atmospheric Aerosols and Nucleation, edited by P. E. Wagner and G. Vali, Lecture Notes in Physics, Vol. 309 (Springer, Berlin, 1988).

${ }^{3}$ Nucleation Phenomena, edited by A. C. Zettlemoyer (Elsevier, Amsterdam, 1977).

${ }^{4}$ F. F. Abraham, Homogeneous Nucleation Theory (Academic, New York, 1974).

${ }^{5}$ R. H. Heist and H. He, J. Phys. Chem. Ref. Data 23, 781 (1995).

${ }^{6}$ R. Strey, P. E. Wagner, and Y. Viisanen, J. Phys. Chem. 98, 7748 (1994).

${ }^{7}$ Y. Viisanen and R. Strey, J. Chem. Phys. 101, 7835 (1994).

${ }^{8}$ Y. Viisanen, R. Strey, and H. Reiss, J. Chem. Phys. 99, 4680 (1993).

${ }^{9}$ D. Wright, R. Caldwell, C. Moxely, and M. S. El-Shall, J. Chem. Phys. 98, 3356 (1993).

${ }^{10}$ D. Wright, R. Caldwell, and M. S. El-Shall, Chem. Phys. Lett. 176, 46 (1991).

${ }^{11}$ M. S. El-Shall, J. Phys. Chem. 93, 8253 (1989).

${ }^{12}$ M. S. El-Shall, J. Chem. Phys. 90, 6533 (1989).

${ }^{13}$ M. S. El-Shall, Chem. Phys. Lett. 143, 381 (1988). 
${ }^{14}$ T. Wimpfheimer, M. A. Chowdhury, and M. S. El-Shall, J. Phys. Chem. 97, 3930 (1993).

${ }^{15}$ C. H. Hung, M. J. Krasnopler, and J. L. Katz, J. Chem. Phys. 90, 1856 (1989).

${ }^{16}$ F. Peters and B. Paikert, J. Chem. Phys. 91, 5672 (1989).

${ }^{17}$ J. Martens, H. Uchtmann, and F. Hensel, J. Phys. Chem. 91, 2489 (1987).

${ }^{18}$ G. W. Adams, J. L. Schmitt, and R. A. Zalabsky, J. Chem. Phys. 81, 5074 (1984).

${ }^{19}$ G. Agarwal and R. H. Heist, J. Chem. Phys. 73, 902 (1980).

${ }^{20}$ C. Becker, H. Reiss and R. H. Heist, J. Chem. Phys. 68, 3585 (1978).

${ }^{21}$ J. L. Katz, P. Mirabel, C. J. Scoppa, and T. L. Virkler, J. Chem. Phys. 65, 382 (1976)

${ }^{22}$ R. H. Heist, K. M. Colling, and C. S. Dupuis, J. Chem. Phys. 65, 5147 (1976).

${ }^{23}$ V. Ruth, J. P. Hirth, and G. M. Pound, J. Chem. Phys. 88, 7079 (1988).

${ }^{24}$ J. L. Katz and M. D. Donohue, Adv. Chem. Phys. 40, 137 (1979).

${ }^{25}$ J. S. Langer and L. A. Turski, Phys. Rev. A 8, 3230 (1973).

${ }^{26}$ J. Lothe and G. M. Pound, J. Chem. Phys. 481849 (1968).

${ }^{27}$ H. Reiss, J. L. Katz, and R. E. Cohen, J. Chem. Phys. 48, 5553 (1968).

${ }^{28}$ H. Reiss and J. L. Katz, J. Chem. Phys. 46, 2496 (1967).

${ }^{29}$ D. W. Oxtoby and R. Evans J. Chem. Phys. 89, 7521 (1988).

${ }^{30}$ A. Dillmann and G. E. A. Meier, Chem. Phys. Lett. 160, 71 (1989).

${ }^{31}$ H. Reiss, A. Tabazabeh and J. Talbot, J. Chem Phys. 92, 1266 (1990)

${ }^{32}$ A. Dillmann and G. E. A. Meier, J. Chem. Phys. 94, 3872 (1991).

${ }^{33}$ H. M. Ellerby, C. L. Weakliem, and H. Reiss, J. Chem Phys. 95, 9209 (1991).

${ }^{34}$ D. W. Oxtoby and X. C. Zeng, J. Chem. Phys. 94, 4472 (1991).

${ }^{35}$ D. W. Oxtoby, J. Phys. Condensed Matter 5, 7627 (1992).

${ }^{36}$ H. M. Ellerby and H. Reiss, J. Chem Phys. 97, 5766 (1992).

${ }^{37}$ C. L. Weakliem and H. Reiss, J. Chem. Phys. 99, 5374 (1993); 101, 2398 (1994).

${ }^{38}$ S. H. Bauer and C. F. Wilcox, Jr., J. Phys. Chem. 97, 11551, 271 (1993).

${ }^{39}$ V. Talanquer and D. W. Oxtoby, J. Chem. Phys. 99, 4670 (1993).

${ }^{40}$ V. Talanquer and D. Oxtoby, J. Chem. Phys. 100, 5190 (1994).

${ }^{41}$ R. Nyquist, V. Talanquer, and D. Oxtoby, J. Chem. Phys. 103, 1175 (1995).

${ }^{42}$ G. Wilemski, J. Chem. Phys. 103, 1119 (1995)

${ }^{43}$ D. W. Oxtoby, in Fundamentals of Inhomogeneous Fluids, edited by D. Henderson (Marcel Dekker, New York 1992), pp. 407-442.

${ }^{44}$ M. Iwamatsu, J. Phys.: Condensed Matter 5, 7537 (1993).

${ }^{45}$ E. N. Fuller, K. Enlet, and J. C. Giddings, J. Chem. Phys. 73, 3679 (1969).

${ }^{46}$ Handbook of Chemistry and Physics, 63rd ed., edited by R. C. Weast (Chemical Rubber Boca Raton, 1982), C-727.

${ }^{47}$ R. C. Reid, J. M. Prausnitz, and E. M. Boling, The Properties of Gases and Liquids, 4th ed. (McGraw-Hill, New York, 1987).

${ }^{48}$ J. J. Jasper, J. Phys. Chem. Ref. Data 1, 841 (1972).

${ }^{49}$ R. H. Heist and H. Reiss, J. Chem. Phys. 59, 15 (1973).
${ }^{50}$ J. L. Katz, J. A. Fisk, and V. Chakarov, in "Nucleation and Atmospheric Aerosols" edited by N. Fukuta and P. E. Wagner (Deepak, Hampton, 1992), p. 11

${ }^{51}$ R. H. Heist, M. Janjua, and J. Ahmed, J. Phys. Chem. 98, 4443 (1994).

${ }^{52}$ R. H. Heist, J. Ahmed, and M. Janjua, J. Phys. Chem. 99, 375 (1995).

${ }^{53}$ P. E. Wagner, R. Strey, and Y. Visanen, in Nucleation and Atmospheric Aerosols, edited by N. Fukuta and P. E. Wagner (Deepak, Hampton, 1992), p. 27.

${ }^{54}$ G. Wilemski, B. E. Wyslouzil, M. Gauthier, and M. B. Frish, in Nucleation and Atmospheric Aerosols, edited by N. Fukuta and P. E. Wagner (Deepak, Hampton, 1992), p. 23.

${ }^{55}$ I. J. Ford, in Nucleation and Atmospheric Aerosols, edited by N. Fukuta and P. E. Wagner (Deepak, Hampton, 1992), p. 39.

${ }^{56}$ I. J. Ford, J. Aerosol Sci. 23, 447 (1992).

${ }^{57}$ D. W. Oxtoby and A. Laaksonen, J. Chem. Phys. 102, 6846 (1995).

${ }^{58}$ D. Kashchiev, J. Chem. Phys. 104, 8671 (1996).

${ }^{59}$ J. A. Fisk and J. L. Katz, J. Chem. Phys. 104, 8649 (1996).

${ }^{60}$ D. Kane, S. P. Fisenko, and M. S. El-Shall (in preparation).

${ }^{61}$ J. L. Schmitt, G. W. Adams, and R. A. Zalabsky, J. Chem. Phys. 77, 2089 (1982).

${ }^{62}$ A. Kacker and R. H. Heist, J. Chem. Phys. 82, 2734 (1985).

${ }^{63}$ R. Strey, T. Schmeling, and P. E. Wagner, J. Chem. Phys. 85, 6192 (1986).

${ }^{64}$ S. M. Thompson, K. Gubbins, J. Walton, R. Chantry, and J. Rowlinson, J. Chem. Phys. 81, 530 (1984).

${ }^{65}$ P. Shreve, J. Walton, and K. Gubbins, J. Chem. Phys. 85, 2178 (1986).

${ }^{66}$ V. Paul Gregory and John C. Schug, Mol. Phys. 78, 407 (1993).

${ }^{67}$ R. C. Tolman, J. Chem. Phys. 17, 118, 333 (1949).

${ }^{68}$ J. G. Kirkwood and F. P. Buff, J. Chem. Phys. 17, 338 (1949).

${ }^{69}$ F. F. Abraham, in Ref. 3, p. 26.

${ }^{70}$ D. Jayaraman, C. Subramanian, and P. Ramasamy in Atmospheric Aerosols and Nucleation, edited by P. E. Wagner and G. Vali (Springer, Berlin, 1988), p. 446.

${ }^{71}$ R. McGraw, J. Chem. Phys. 75, 5514, (1981).

${ }^{72}$ B. N. Hale, Phys. Rev. A 33, 4156 (1986).

${ }^{73}$ B. N. Hale, in Scaled Models for Nucleation, Lecture Notes in Physics, Vol. 309, edited by P. E. Wagner and G. Vali (Springer, New York, 1988), pp. 323-349.

${ }^{74}$ B. N. Hale and P. Kemper, J. Chem. Phys. 91, 4314 (1989).

${ }^{75}$ B. N. Hale and B. Kelly, Chem. Phys. Lett. 189, 100 (1992).

${ }^{76}$ S. L. Girshick and C. P. Chiu, J. Chem. Phys. 93, 1273 (1990).

${ }^{77}$ V. I. Kalikmanov and M. E. H. van Dongen, Phys. Rev. E 51, 4391 (1995).

${ }^{78}$ L. Granasy, J. Chem. Phys. 104, 5188 (1996).

${ }^{79}$ L. Root and F. Stillinger, J. Chem. Phys. 90, 1200 (1989).

${ }^{80}$ D. Wright and M. S. El-Shall, J. Chem. Phys. 100, 3791 (1994). 